\title{
Optical and Ultraviolet Observations of the Very Young Type IIP SN 2014cx in NGC 337
}

\author{
Fang Huang ${ }^{1}$, Xiaofeng Wang ${ }^{1}$, Luca Zampieri² ${ }^{2}$, Maria Letizia Pumo ${ }^{3}$, Iair Arcavi ${ }^{4,5}$, Peter \\ J. Brown ${ }^{6}$, Melissa L. Graham ${ }^{7}$, Alexei V. Filippenko ${ }^{7}$, WeiKang Zheng ${ }^{7}$, Griffin \\ Hosseinzadeh $^{4}$, D. Andrew Howell ${ }^{4,8}$, Curtis $\mathrm{McCully}^{4}$, Liming Rui ${ }^{1}$, Stefano Valenti ${ }^{9}$, \\ Tianmeng Zhang ${ }^{10}$, Jujia Zhang ${ }^{11,12}$, Kaicheng Zhang ${ }^{1}$, Lifan Wang ${ }^{6}$
}


Received

accepted

\footnotetext{
${ }^{1}$ Physics Department and Tsinghua Center for Astrophysics, Tsinghua University, Beijing, 100084, China; huangfang@mail.tsinghua.edu.cn; wang_xf@mail.tsinghua.edu.cn

${ }^{2}$ INAF-Osservatorio Astronomico di Padova, Vicolo dell'Osservatorio 5, 35122 Padova, Italy
}

${ }^{3}$ INAF-Osservatorio Astronomico di Palermo "Giuseppe S. Vaiana," Piazza del Parlamento 1, 90134 Palermo, Italy

${ }^{4}$ Las Cumbres Observatory Global Telescope Network, 6740 Cortona Dr., Suite 102, Goleta, CA 93117, USA

${ }^{5}$ Kavli Institute for Theoretical Physics, Kohn Hall, University of California, Santa Barbara, CA 93106-4030, USA

${ }^{6}$ George P. and Cynthia Woods Mitchell Institute for Fundamental Physics \& Astronomy, Texas A\&M University, Department of Physics and Astronomy, 4242 TAMU, College Station, TX 77843 , USA

${ }^{7}$ Department of Astronomy, University of California, Berkeley, CA 94720-3411, USA

${ }^{8}$ Department of Physics, University of California, Santa Barbara, Broida Hall, Mail Code 9530, Santa Barbara, CA 93106-9530, USA

${ }^{9}$ Department of Physics, University of California, Davis, CA 95616, USA

${ }^{10}$ Key Laboratory of Optical Astronomy, National Astronomical Observatories, Chinese Academy of Sciences, Beijing 100012, China

${ }^{11}$ Yunnan Observatories, Chinese Academy of Sciences, Kunming 650011, China

${ }^{12}$ Key Laboratory for the Structure and Evolution of Celestial Objects, Chinese Academy of Sciences, Kunming 650216, China 


\begin{abstract}
Extensive photometric and spectroscopic observations are presented for SN 2014cx, a type IIP supernova (SN) exploding in the nearby galaxy NGC 337. The observations are performed in optical and ultraviolet bands, covering from -20 to +400 days from the peak light. The stringent detection limit from prediscovery images suggests that this supernova was actually detected within about 1 day after explosion. Evolution of the very early-time light curve of SN 2014cx is similar to that predicted from a shock breakout and post-shock cooling decline before reaching the optical peak. Our photometric observations show that SN 2014cx has a plateau duration of $\sim 100$ days, an absolute $V$-band magnitude of $\sim-16.5 \mathrm{mag}$ at $t \approx 50$ days, and a nickel mass of $0.056 \pm 0.008 \mathrm{M}_{\odot}$. The spectral evolution of SN 2014cx resembles that of normal SNe IIP like SN 1999em and SN 2004et, except that it has a slightly higher expansion velocity $\left(\sim 4200 \mathrm{~km} \mathrm{~s}^{-1}\right.$ at 50 days). From the cooling curve of photospheric temperature, we derive that the progenitor has a pre-explosion radius of $\sim 640 \mathrm{R}_{\odot}$, consistent with those obtained from SNEC modeling $\left(\sim 620 \mathrm{R}_{\odot}\right)$ and hydrodynamical modeling of the observables $\left(\sim 570 \mathrm{R}_{\odot}\right)$. Moreover, the hydrodynamical simulations yield a total explosion energy of $\sim 0.4 \times 10^{51} \mathrm{erg}$, and an ejected mass of $\sim 8 \mathrm{M}_{\odot}$. These results indicate that the immediate progenitor of SN 2014cx is likely a red supergiant star with a mass of $\sim 10 \mathrm{M}_{\odot}$.
\end{abstract}

Subject headings: supernovae: general - supernovae: individual: SN 2014cx galaxies: individual: NGC 337 


\section{Introduction}

Type IIP supernovae (SNe IIP) represent the most common subtype of stellar explosions, constituting about one third of all SNe ( $\mathrm{Li}$ et al. 2011). This subtype of SNe is thought to arise from core-collapse (CC) explosions of massive red supergiants (RSGs) with an initial mass of 8-25 $\mathrm{M}_{\odot}$ according to theoretical models of stellar evolution (Heger et al. 2003). On the other hand, direct analyses of supernova position on pre-explosion images give a much narrower range for the progenitor mass, e.g., 8.5-16.5 $\mathrm{M}_{\odot}$ (Li et al. 2007) Smartt 2009). Compared to other CC SNe, SNe IIP are characterized by prominent hydrogen features in their optical spectra (e.g., Filippenko 1997) and an extended plateau phase in their light curves. During the plateau phase, their luminosity remains almost constant as a result of the energy balance between the hydrogen recombination and expansion cooling. The plateau feature distinguishes SNe IIP from the Type IIL subclass, for which the light curve exhibits a linear decline (in mag day ${ }^{-1}$ ) after the peak (Barbon et al. 1979). Recent statistical analyses show that the light-curve properties of SNe II may have a continuous distribution (e.g., Anderson et al. 2014; Sanders et al. 2015; Valenti et al. 2016), although there are also studies suggesting a distinct division between type IIP and IIL SNe (Arcavi et al. 2012; Faran et al. 2014a, b).

Over the years, numerous SNe IIP have been well studied, such as SN 1999em (Leonard et al. 2002), SN 2004et (Sahu et al. 2006), SN 2005cs (Pastorello et al. 2009), and SN 2013ej (Huang et al. 2015). These studies reveal a large spread in luminosities, plateau durations, expansion velocities, and nickel masses for SNe IIP (e.g., Hamuv 2003), which can be well understood with current explosion models (e.g., Kasen \& Woosley 2009; Dessart et al. 2010; Pumo \& Zampieri 2011, 2013). Nevertheless, early time observations are still sparse for SNe IIP, which are vital to constrain the explosion time and hence determine the properties of their progenitor stars (Nadyozhin 2003; Smartt et al. 2009). In 
particular, very early light curves of SNe IIP may be affected by a short, sharp blast of light as a result of shock breakout of the stellar surface, as predicted in core collapse explosion of massive stars (Falk \& Arnett 1977; Klein \& Chevalier 1978). SN 2014cx represents such a CC SN that is captured within about one day after the explosion.

SN 2014cx was independently discovered on UT 2014 Sept. 2 by Nakano et al. (2014) and Holoien et al. (2014) in the nearby SBd galaxy NGC 337. Based on the astrometry from the USNO-A2.0 Catalogue (Monet 1998), the J2000 coordinates of the SN are derived as $\alpha=00^{h} 59^{m} 47.83^{s}$ and $\delta=-07^{\circ} 34^{\prime} 19.3^{\prime \prime}$, approximately $21.7^{\prime \prime} \mathrm{N}$ and $33.7^{\prime \prime} \mathrm{W}$ from the center of NGC 337 (Holoien et al. 2014). SN 2014cx was reported as a young SN II based on both optical (Elias-Rosa et al. 2014) and near-infrared (NIR; Morrell et al. 2014) spectra taken at about one day after the discovery. It was further classified as a Type IIP event according to the photometric observations by Andrews et al. (2015). We note that another SN IIP, SN 2011dq, also exploded in NGC 337. The distance to NGC 337 is estimated to be $18.0 \pm 3.6 \mathrm{Mpc}$ (distance modulus $\mu=31.27 \pm 0.43 \mathrm{mag}$ ) by the Tully-Fisher method (Sorce et al. 2014); here we adopt this value for SN 2014cx.

In this work, we present the results of our optical and UV observations of the type IIP supernova SN 2014cx that was discovered at a very young age. The observations and data reduction are addressed in Section 2, the photometric and spectroscopic evolution are described in Section 3 and 4, respectively, and analysis of the progenitor properties of SN 2014cx via the photospheric temperature cooling curve and hydrodynamical modeling is given in Section 5. The main results are summarized in Section 6. 


\section{Observations and Data Reduction}

\subsection{Photometry}

\subsubsection{Optical Observations}

We started the $U B V R I$ follow-up campaign with the $0.8 \mathrm{~m}$ Tsinghua University-NAOC telescope (hereafter TNT) at Xinglong Observatory in China (Wang et al. 2008; Huang et al. 2012). The TNT monitoring of SN 2014cx started on 2014 Sep. 15 and continued until 2015 Jan. 31. High-cadence Johnson BV and Sloan gri monitoring was conducted using the $1.0 \mathrm{~m}$ telescopes in the Las Cumbres Observatory Global Telescope network (hereafter LCOGT; Brown et al. 2013). The LCOGT data cover 2014 Sep. 3 through 2015 Sep. 17. In addition, 55 epochs of unfiltered data were collected with the $0.76 \mathrm{~m}$ Katzman Automatic Imaging Telescope (KAIT; Filippenko et al. 2001), extending to 2015 Jan. 5.

All of the images were reduced with standard IRAF1 routines. Point-spread function (PSF) photometry was performed on TNT and LCOGT data using the SNOOPY package2. KAIT data were reduced using a PSF-based image-reduction pipeline (Ganeshalingam et al. 2010). The SN instrumental magnitudes were calibrated using 15 field stars (marked in Figure 1) from the Sloan Digital Sky Survey (SDSS) Data Release 9 catalog (Ahn et al. 2012) and transformed to the Johnson system. The magnitudes of the reference stars are listed in Table 1. The final flux-calibrated optical magnitudes of SN 2014cx are shown in Table 2 4 .

\footnotetext{
${ }^{1}$ IRAF is distributed by the National Optical Astronomy Observatories, which are operated by the Association of Universities for Research in Astronomy, Inc., under cooperative agreement with the National Science Foundation (NSF).

${ }^{2}$ http://sngroup.oapd.inaf.it/snoopy.html
} 


\subsubsection{Swift Ultraviolet Observations}

In addition to the ground-based observations, SN 2014cx was also monitored with the Ultraviolet/Optical Telescope (UVOT; Roming et al. 2005) on board the Swift spacecraft through the uvw2, uvm2, uvw1, u,b, and $v$ filters. The UV observations began on 2014 Sep. 3 and ended on 2014 Oct. 24. The data were obtained from the Swift Optical/Ultraviolet Supernova Archive (SOUSA; Brown et al. 2014). The reduction method for UVOT photometry is based on that of Brown et al. (2009), which includes aperture photometry after subtracting off the underlying galaxy count rates, and adopting the updated zeropoints and time-dependent sensitivity from Breeveld et al. (2011). The final UVOT photometry of SN 2014cx is presented in Table 5 .

\subsection{Spectroscopy}

The spectroscopic monitoring campaign of SN 2014cx started on 2014 Sep. 7 and continued for $\sim 400$ days. Fourteen low-resolution optical spectra were obtained using the LCOGT 2 m Faulkes Telescope South (FTS; with FLOYDS), the Xinglong 2.16 m telescope (with BFOSC), the Lijiang $2.4 \mathrm{~m}$ telescope (with YFOSC), the LCOGT $2 \mathrm{~m}$ Faulkes Telescope North (FTN; with FLOYDS), the GEMINI North Telescope (GNT; with GMOS), and the Keck-I $10 \mathrm{~m}$ telescope (with LRIS); see Table 7 for the detailed information on the spectroscopic observations.

The spectra were all reduced in a standard manner using various tasks within IRAF. After the preliminary reduction (including bias/overscan correction, flat fielding, and cosmic-ray removal), the one-dimensional spectra were extracted using the optimal extraction method (Horne 1986). Wavelengths of the SN spectra were calibrated using the $\mathrm{Fe} / \mathrm{Ar}$ and $\mathrm{Fe} / \mathrm{Ne}$ lamp spectra. For the FLOYDS spectra, a $\mathrm{Hg} / \mathrm{Ar}$ lamp is used 
for wavelength calibration. The spectra were then flux calibrated using the instrumental sensitivity curves of spectrophotometric standard stars observed on the same (or nearby) night and with the same instrumental setup.

\section{Photometric Analysis}

\subsection{The Early-Time Evolution}

With modern high-cadence surveys, we are able to detect young SNe and trigger follow-up observations immediately. Such early-time detections help constrain the explosion properties and (with luck) even reveal shock breakout, which often happens hours after explosion (Quimby et al. 2007; Schawinski et al.|2008; Tominaga et al. 2009; Gezari et al. 2015; Garnavich et al. 2016; Dhungana et al. 2016).

The field of SN 2014cx was monitored daily by KAIT in the clear band (i.e., unfiltered) before the explosion, leading to the earliest detection of this object after the explosion. Figure 2 shows the KAIT unfiltered light curve, spanning from a few weeks before the explosion to $\sim 120$ days after. A few early-time observations obtained by amateurs in unfiltered mode are also overplotted. As can be seen, SN 2014cx was not detected on 2014 Sep. 1 (MJD = 56,901.39) with a limit of > $19.1 \mathrm{mag}$, but it was detected one day later $(\mathrm{MJD}=56,902.40)$ at $15.69 \mathrm{mag}$. We therefore adopt this epoch $(\mathrm{MJD}=56901.89 \pm 0.5)$ as the reference date for the shock breakout for SN 2014cx, and this determination sets one of the tightest constraints on the shock breakout time for a SN IIP based on the observations of ground-based telescopes.

The unfiltered light curve of SN 2014cx at early time (0-20 days) is very similar to those

of SN 2006bp and SN 2013ej. It seems to experience three stages. First, the SN brightened by more than 4 mag over the first 1.5-2.0 days, and it then dimmed and rebrightened 
toward the primary peak at $t \approx 20$ days. This trend is actually more prominent in the early-time evolution of the LCOGT $r$-band light curve (see Figure 2), with a noticeable dip occurring at $\mathrm{t} \approx 10$ days after explosion. Such a behavior is also seen in the comparison object SN 2006bp (and possibly SN 2013ej), which is likely related to the shock breakout of the supernova surface and its cooling phase (Quimby et al. 2007).

\subsection{Overall Evolution of the Light Curves}

Figure 3 shows the multicolor photometric evolution of SN 2014cx during the period from $t=+1$ to $t=+380$ days after the explosion. At the earliest phases, the light curves exhibit a rapid rise in the UV and optical bands, but with a slower pace at longer wavelengths (see Table 6). By fitting a low-order polynomial to the data around maximum light, we determine the magnitudes and corresponding dates at peak brightness. These results are also presented in Table 6. We note that the rise time in the $B$ band is $\sim 8$ days, which agrees well with the statistical result from a large sample of SNe II (González-Gaitán et al. 2015).

One can see from Figure 3 that the UV light curves show rapid post-peak declines, with slopes of $0.16,0.23$, and $0.25 \mathrm{mag}^{-1}$ in the uvw1, uvw2, and uvm2 bands, respectively. The optical light curves, in contrast, drop slowly and settle to the plateau phase. A decline rate of $\beta_{100}^{B}=2.5 \mathrm{mag}$ is measured for the $B$-band light curve over the first $100 \mathrm{~d}$ after maximum light, consistent with the typical value obtained for normal SNe IIP (i.e., $\beta_{100}^{B}<3.5$ mag; Patat et al. 1994). The $V$-band brightness declines by $\sim 0.2 \mathrm{mag}$ in the first 50 days after the peak, which also falls into the SN IIP group according to the criterion of Faran et al. (2014b). For SN 2014cx, the plateau phase lasts for $\sim 100$ days, and its brightness stays roughly constant, especially in the $\operatorname{Vr} R i I$ bands. 
After the plateau, the SN 2014cx experiences a sudden drop in brightness in all optical bands, with magnitude declines of 1.5-2.0 mag in $\sim 20$ days. To derive the parameters about the transitional phase, we fit the $V$-band light curve using Eq.(4) from Olivares et al. (2010). The middle date of the transition phase, $t_{\mathrm{PT}}$, is found to be $\sim 109 \mathrm{~d}$, and the width of the transition phase is $\sim 7 \mathrm{~d}$. During the tail phase (i.e., $t \approx 130-170$ days), the decline rates in $B, V, R$, and $I$ are measured to be $0.42,0.95,1.09$, and $1.13 \mathrm{mag}(100 \mathrm{~d})^{-1}$, respectively. These values are similar to those of normal SNe IIP such as SN 1999em (Elmhamdi et al. 2003). In the $V$ and $R$ bands, the decline rates are found to be comparable to the values expected for the radioactive decay, $0.98 \mathrm{mag}(100 \mathrm{~d})^{-1}$ (Patat et al. 1994). This indicates that the gamma-ray photons were effectively trapped in the ejecta at this phase. After $t \approx 170$ days, the light curves tend to show slower decline rates relative to the earlier nebular phase. For example, the decline rate of $B$ - and $V$-band light curve is measured to be 0.32 and $0.65 \mathrm{mag}(100 \mathrm{~d})^{-1}$ during the period from 170 days $<\mathrm{t}<320$ days. And this decay rate is found to be $0.46,0.85$, and $0.81 \mathrm{mag}(100 \mathrm{~d})^{-1}$ in gri bands at similar phase. This slower evolution can be due to interaction of SN ejecta with the circumstellar materials surrounding the SN or a scattered light echo (see discussions in $\S 4.1)$.

\subsection{Color Curves}

The Galactic reddening in the direction of NGC 337 is $E(B-V)_{\mathrm{MW}}=0.10 \mathrm{mag}$ (Schlafly \& Finkbeiner 2011). We didn't detect any significant features of Na I D absorption produced by the host galaxy in our 14 low-resolution spectra, suggesting that host-galaxy reddening might be negligible for SN 2014cx. As an alternative, we also use the color method proposed by Olivares et al. (2010) to estimate the reddening due to the host galaxy. This method assumes that the intrinsic $V-I$ color of SNe IIP is constant (i.e., 
$\left.(V-I)_{0}=0.656 \mathrm{mag}\right)$ toward the end of the plateau. After correcting for the Galactic reddening, the $V-I$ color of SN 2014cx is found to be 0.596 mag at $t=79$ days, which gives a negative host-galaxy extinction for this object. Thus, we adopt $E(B-V)_{\text {tot }}=0.10$ mag in the following analysis, which corresponds to a total line-of-sight extinction of $A_{V}=$

$0.31 \mathrm{mag}$ assuming the ratio of total to selective extinction $R_{V}=3.1$ (Cardelli et al. 1989).

The reddening-corrected $(U-B)_{0},(B-V)_{0},(V-R)_{0}$, and $(V-I)_{0}$ color curves of SN 2014cx are shown in Figure 4. For comparison, we overplot the dereddened color curves of three well-studied type IIP SNe 1999em, 2004et, and 2005cs. The color evolution of SN 2014cx follows the general trend seen in SNe IIP: a rapid decrease from blue (high temperature) to red (low temperature) during the photospheric phase. The $(U-B)_{0}$ and $(B-V)_{0}$ colors become redder by $\sim 1-2$ mag in the first 100 days, while the $(V-R)_{0}$ and $(V-I)_{0}$ colors evolve slowly and become red by only $\sim 0.5$ mag. The $(B-V)_{0}$ color turns blue after $\mathrm{t} \sim 120 \mathrm{~d}$, when the nebular phase begins. The overall colors of SN 2014cx are bluer than those of SN 1999em at all phases, except in $V-I$ where these two objects have similar colors. This suggests that SN 2014cx has a higher photospheric temperature than SN 1999em.

\subsection{Bolometric Light Curve}

To derive the bolometric luminosity of SN 2014cx , we first convert the extinctioncorrected magnitudes in different bands to flux values at the effective wavelength, and then integrate the combined flux over wavelength. The luminosity can then be calculated using the integrated flux and a distance of $18 \mathrm{Mpc}$ (see Section 2). During the first 50 days, when Swift UV photometry was available, we integrated the UV flux and extrapolated it to the optical flux. As the UV flux decreases quickly, we assume that the UV contribution to the bolometric flux is marginal in the late plateau phase and negligible $(\lesssim 1 \%)$ during the 
nebular phase (e.g., Dall'Ora et al. 2014). Owing to the lack of NIR data, we estimate the tail luminosity using Equation 3 in Maguire et al. (2010), where a bolometric correction of $0.33 \pm 0.06 \mathrm{mag}$ is adopted.

Figure 5 shows the UV-optical-NIR ("UVOIR") bolometric luminosity curve of SN 2014cx. One can see that it reached a peak of log $\left[L_{\mathrm{bol}}^{\text {peak }} /\left(\operatorname{erg~s}^{-1}\right)\right]=42.47$ at $\sim 3 \mathrm{~d}$ after explosion. In Figure 5 we also compare the $U B V R I$ quasi-bolometric luminosity of SN 2014cx with that of some well-studied SNe IIP. It is readily seen that the luminosity evolution of SN 2014cx is similar to that of SN 2004et and SN 1999em but lies between these two objects in the early and plateau phases. In the nebular phase, we note that the tail luminosity of SN 2014cx is apparently higher than SN 1999em and even slightly higher than SN 2004et, suggesting that a relatively larger amount of nickel may be synthesized in its explosion (see discussion below).

\section{5. $\quad{ }^{56} \mathrm{Ni}$ Mass}

For SNe IIP, the light curve in the nebular phase is powered mainly by the radioactive decay chain ${ }^{56} \mathrm{Ni} \rightarrow{ }^{56} \mathrm{Co} \rightarrow{ }^{56} \mathrm{Fe}$. And the tail luminosity is directly proportional to the mass of synthesized ${ }^{56} \mathrm{Ni}$ supposing that the gamma-ray deposition fraction is similar. For the well-studied type II-pec SN 1987A, the mass of ${ }^{56} \mathrm{Ni}$ has been determined to be $0.075 \pm 0.005 \mathrm{M}_{\odot}($ Arnett 1996). For SN 2014cx we estimate the UVOIR bolometric luminosity at $\mathrm{t} \approx 150 \mathrm{~d}$ to be $1.93 \pm 0.14 \times 10^{41} \mathrm{erg} \mathrm{s}^{-1}$ by making a linear fit to the evolution between $\mathrm{t} \approx 120 \mathrm{~d}$ and $\mathrm{t} \approx 200 \mathrm{~d}$. The luminosity of SN 1987A at the same phase is estimated to be $2.47 \pm 0.02 \times 10^{41} \mathrm{erg} \mathrm{s}^{-1}$. The ratio of SN 2014cx to SN 1987A is $0.78 \pm 0.06$, which yields a value of $M_{\mathrm{Ni}}=0.058 \pm 0.006 \mathrm{M}_{\odot}$ for $\mathrm{SN} 2014 \mathrm{cx}$.

Based on the assumption that all $\gamma$-rays from ${ }^{56} \mathrm{Co} \rightarrow{ }^{56} \mathrm{Fe}$ are entirely thermalized, 
Hamuy (2003) found an independent relationship to estimate the value of ${ }^{56} \mathrm{Ni}$ mass using the tail luminosity $\left(L_{\mathrm{t}}\right)$. We calculated $L_{\mathrm{t}}$ of SN $2014 \mathrm{cx}$ at 17 epochs from $\mathrm{t} \approx 120$ days to $\approx 170$ days using the late-time $V$-band magnitude. The mean value of $M_{\mathrm{Ni}}$ resulting from Equation 2 of Hamuy $(2003)$ is $0.055 \pm 0.011 \mathrm{M}_{\odot}$, consistent with the result from direct comparison with SN 1987A.

Following the procedures of Elmhamdi et al. (2003), we also estimated the ${ }^{56} \mathrm{Ni}$ mass using the steepness parameter $S$, which is defined as the steepest value in the $V$-band light curve at the transitional phase from plateau to nebular tail. For SN 2014cx, we obtain $S=0.071 \mathrm{mag} \mathrm{d}^{-1}$ and $M_{\mathrm{Ni}}=0.056 \pm 0.007 \mathrm{M}_{\odot}$, in good agreement with the values derived from the observed luminosity in the radioactive tail. The weighted mean value of $M_{\mathrm{Ni}}$ derived from above three results is $0.056 \pm 0.008 \mathrm{M}_{\odot}$.

\section{Spectroscopic Analysis}

\subsection{Optical Spectra}

The overall spectroscopic evolution of SN 2014cx is displayed in Figures [6 and 7, covering early time $(\sim+6 \mathrm{~d})$ up to the nebular phase $(\sim+404 \mathrm{~d})$. All of the spectra have been corrected for the recession velocity of the host galaxy $\left(1646 \mathrm{~km} \mathrm{~s}^{-1}\right)$ but not for the reddening. The main spectral features are labeled according to the lines previously identified for SNe IIP (Leonard et al. 2002).

At $t \approx 6$ days, the spectrum is very blue with a blackbody temperature exceeding $10^{4} \mathrm{~K}$. The prominent features at such early phases are Balmer lines and He I $\lambda 5876$ with broad P-Cygni profiles. At $t \approx 17$ days, the Fe II $\lambda 5169$ absorption feature is visible, and it becomes stronger by $t \approx 21$ days along with Fe II $\lambda 5018$. The $\mathrm{Na}$ I and He I lines are also detectable at $t \approx 21$ days, and both features grow stronger thereafter. The continuum 
becomes notably redder from $t \approx 21$ days to $t \approx 25$ days, suggesting a rapid decrease of the photospheric temperature during this period. Consequently, more metal lines (such as Sc II, Ba II, O I, and the Ca II NIR triplet) are formed, and they gradually become the dominant features in the spectra.

At $t \approx 128$ days, SN 2014cx starts to enter the nebular phase. The spectrum shows deep absorption of $\mathrm{Na} \mathrm{I}$ and prominent emission lines of [O I] $\lambda \lambda 6300,6364$, [Fe II] $\lambda \lambda 7155$, and $[\mathrm{Ca}$ II] $\lambda \lambda 7291,7324$. At $t \approx 329$ days and $t \approx 404$ days, the spectra exhibit emission lines of $\mathrm{H} \alpha,[\mathrm{O} \mathrm{I}],[\mathrm{Fe} \mathrm{II}]$, and [Ca II], as well as P-Cygni profiles of Na I, O I, Fe II, and the Ca II NIR triplet. The multiple emission peaks at 5000-5500 $\AA$ are likely contributed by [Fe I] and [Fe II] multiplets. We note that the continuum at 3800-5000 is relatively bluer than that taken at $128 \mathrm{~d}$, perhaps because of either a scattered light echo off the surrounding circumstellar material (CSM; Andrews et al. 2016) or late-time CSM interaction (Inserra et al. 2011). For the light-echo scenario, the echoed light should come from earlier phases when the spectra were blue, and the scattering is more efficient at shorter wavelengths. However, the negligible extinction estimated for SN 2014cx in Section 3.3 implies that the CSM potentially causing the light echoes is in the opposite direction of SN 2014cx. In the case of CSM interaction, we can also see a hint of narrow $\mathrm{H} \alpha$ emission in the spectrum at $\mathrm{t} \approx 404$ days.

In Figure 8, we compare the spectra of SN 2014cx at about one week, 50 days, and one year after explosion with those of SN 1999em, SN 2005cs, and SN 2004et at similar phases. It can be seen that SN 2014cx has relatively shallower line profiles than the comparison SNe at early phases. During the plateau phase, $t \approx 50$ days after the explosion, the hydrogen lines of SN 2014cx are similar to those of the comparison SNe IIP, but stronger than those of the subluminous SN 2005cs. In the nebular phase $(t \approx 300$ days), however, SN 2014cx appears to have weaker spectral features than SN 1999em and SN 2004et, but stronger than 
SN 2005cs, consistent with a flux contribution to the continuum.

\subsection{Expansion Velocities}

We measured the relativistic Doppler velocities of the $\mathrm{H} \alpha, \mathrm{H} \beta$, Fe II $\lambda 5169$, and Sc II $\lambda 6245$ lines during the photospheric phase by fitting a Gaussian function to their absorption minima. The velocity evolution of these ions is shown in Figure 9. One can see that the velocities of hydrogen lines are higher than those of metal lines; the hydrogen lines have a lower optical depth and thus are formed at larger radii in the ejecta. The velocities seem to decline with an exponential trend.

To examine the differences of photospheric velocity between SN 2014cx and other SNe IIP, we compared the velocity as measured from Fe II $\lambda 5169$ line. As shown in Figure 10, SN 2014cx and SN 2014et have similar velocities at comparable phases, and their expansion velocities are higher than that of SN 1999em (by $\sim 1000 \mathrm{~km} \mathrm{~s}^{-1}$ ) and SN 2005cs (by $~ 3000$ $\left.\mathrm{km} \mathrm{s}^{-1}\right)$.

\section{Progenitor Estimates}

\subsection{Radius of Progenitor}

For CC SNe, following the shock breakout, the heated stellar envelope expands and then cools down. The different timescales of photospheric temperature evolution depend mainly upon the initial radius of the progenitor and the opacity. A simple analytic function has been proposed by Rabinak \& Waxman (2011) to estimate the radius of the progenitor of CC SNe using the early-time temperature evolution. Theoretically, the duration that the photosphere can stay at a higher temperature depends on the radius of progenitor star, 
with longer time for a progenitor with a larger radius (and vice versa).

We constructed the spectral energy distribution (SED) and computed the blackbody temperatures using the Swift UV and optical data obtained during the first week after explosion. The luminosity and temperature can then be used to constrain the radius of the progenitor star by using Equation 13 of Rabinak \& Waxman (2011) (e.g., Valenti et al. 2014; Bose et al. 2015; Rubin et al. 2016). Note that the equation is only valid for the first week after explosion, when the light curve is dominated by shock cooling at very early phase, and the photosphere is located at the outer shell of the expanding ejecta (Rubin et al. 2016). Adopting an optical opacity of $0.34 \mathrm{~cm}^{2} \mathrm{~g}^{-1}$ and a typical RSG density profile $f_{\rho}=0.13$, we obtained an initial radius of $643 \pm 60 \mathrm{R}_{\odot}$ for the progenitor of $\mathrm{SN}$ 2014cx (see Figure 11), consistent with the typical size of an RSG.

Based on the SuperNova Explosion Code (SNEC, Morozova et al.|2015), Morozova et al. (2016) suggests that the early-time evolution of the light curves of SNe IIP relies sensitively on the radius of the exploding star, as explained with an analytical correlation between the $g$-band rise time and the progenitor radius (i.e., $\log R\left[R_{\odot}\right]=1.225 \log t_{\text {rise }}[$ day $]+1.692$ ). Thus, we fit the early-time $g$-band light curve of SN 2014cx and obtain the rise time as $t_{\text {rise }}=7.90 \pm 0.10$ days. Inserting this value into the rise time-radius relation, we estimate the radius of the progenitor to be $619 \pm 10 \mathrm{R}_{\odot}$.

\subsection{Hydrodynamical Modeling}

In this subsection, we further determine the main physical parameters of SN 2014cx and its progenitor (i.e., the explosion energy, the radius of progenitor star, and the ejected mass) using a method of hydrodynamical modeling. This method uses the SN observables (i.e., the bolometric light curve, the velocity evolution, and the temperature of the 
continuum obtained during the photospheric phase) as input parameters to constrain the physical properties of expanding ejecta and the evolution of SN observables (from the shock breakout up to the nebular phase) using the general-relativistic, radiation-hydrodynamics code. This technique has been successfully applied to the studies of numerous SNe IIP, including SNe 2007od, 2009bw, 2009E, 2012A, 2013ab, and 2013ej (Inserra et al.|2011, 2012; Pastorello et al. 2012; Tomasella et al. 2013; Bose et al. 2015; Huang et al. 2015). Details about this hydro-dynamical model are well described in Zampieri et al. (2003); Pumo et al. (2010); Pumo \& Zampieri (2011).

Based on the estimates of explosion epoch (MJD = 56,901.89; Section 3.1), bolometric luminosity, and nickel mass $\left(0.056 \mathrm{M}_{\odot}\right.$; Section 3.5$)$, the best-fit hydrodynamic model returns a total (kinetic plus thermal) energy of $0.4 \times 10^{51} \mathrm{erg}$, an initial radius of $4 \times 10^{13}$ $\mathrm{cm}\left(\sim 570 \mathrm{R}_{\odot}\right)$, and an envelope mass of $8 \mathrm{M}_{\odot}$ (see Figure 12) for SN 2014cx. Considering a mass of $\sim 1.5-2.0 \mathrm{M}_{\odot}$ for the compact remnant star, we estimate that SN 2014cx has an immediate progenitor mass of $9.5-10.0 \mathrm{M}_{\odot}$ when exploding. The zero-age main-sequence mass should be slightly higher given that the progenitor star suffers some mass loss during the lifetime. These values are consistent with those of a typical RSG with relatively low mass. The radius is also in agreement with that estimated from the early temperature as described in Section 5.1 within uncertainties.

We further compare our light curves and spectra with those from Kasen \& Woosley (2009), and we found that our parameters generally fall into the range of their Table 2, except that we have a smaller nickel mass $\left(0.056 \mathrm{M}_{\odot}\right.$ vs. $\left.0.1-0.5 \mathrm{M}_{\odot}\right)$. This difference is likely due to that their numerical models use larger main sequence masses $\left(12-25 \mathrm{M}_{\odot}\right)$ than our hydrodynamic modeling results $\left(9.5-10.0 \mathrm{M}_{\odot}\right)$.

The evolutions of three observables (luminosity, velocity, and temperature) and the modeling results are shown in Figure 12, As it can be seen, the modeling reasonably match 
the observed luminosity/temperature except at early phases where a larger deviation is seen in luminosity. This deviation is likely due to that the density profile in the radial direction of the outermost ejecta cannot be well produced by our simulations (Pumo \& Zampieri 2011). In the middle panel of Figure 12, we note that there is also a small $(\lesssim 10-15 \%)$ discrepancy between our best-fit model and the observed photospheric velocity. This discrepancy may be attributed to a systematic shift between the true photospheric velocity and the values estimated from the observed P-Cygni line profiles (Dessart \& Hillier 2005). This can be explained with the fact that the optical depth is higher for spectral lines relative to the continuum, which can place the line photosphere at a larger radius (see also Inserra et al. 2013).

\section{Discussion and Summary}

In this paper, we present extensive UV and optical photometry and optical spectroscopy of SN 2014cx in NGC 337, spanning the period from -30 d to $+404 \mathrm{~d}$ from the maximum light. The explosion time is constrained to be MJD $=56,901.89$ with an accuracy of \pm 0.5 day.

The characteristics of the light curves, such as the rise time, duration of the plateau phase, post-peak decline, and bolometric luminosity, suggest that SN 2014cx is a normal type IIP supernova. The KAIT unfiltered and LCOGT r-band light curves seem to experience two brightening components, with the first likely related to shock breakout of the supernova. The plateau duration is $\sim 100$ days, similar to that of our comparison SNe IIP. The value of $M_{V}$ at mid-plateau phase $(\sim 50 \mathrm{~d})$ is $-16.48 \pm 0.43 \mathrm{mag}$ for SN 2014cx, lying between the luminous SNe IIP ( -17 mag, SN 2004et) and subluminous SNe IIP ( -15

mag, SN 2005cs). The mass of ${ }^{56} \mathrm{Ni}$ using the tail luminosity and steepness methods yield a value of $0.056 \mathrm{M}_{\odot}$, similar to that of SN $1999 \mathrm{em}$ and SN 2004et. 
The spectroscopic evolution of SN 2014cx shares a similarity with the typical Type IIP SNe 1999em and 2004et. The early-time spectra exhibit a nearly featureless continuum with only hydrogen Balmer lines and He I visible. As the SN evolves, the continuum becomes redder and the metal lines emerge, becoming the dominant features during the photospheric phase. During the nebular phase, the spectra are dominated by strong emission lines. The continuum at $3800-5000 \AA$ is relatively blue, which might be caused by either late-time CSM interaction or a scattered-light echo. The value and evolution of the expansion velocity derived from Fe II $\lambda 5169$ are similar to those of SN 2004et, but $\sim 1000 \mathrm{~km} \mathrm{~s}^{-1}$ higher than the expansion velocity of SN 1999em.

By modeling the observables of SN 2014cx as derived from our observations, we estimate that this explosion produces a total energy of $0.4 \times 10^{51}$ ergs and an ejected mass of $\sim 8.0 \mathrm{M}_{\odot}$. The progenitor star is calculated to have a radius of $4 \times 10^{13} \mathrm{~cm}\left(\sim 574 \mathrm{R}_{\odot}\right)$, which agrees well with that estimated from the early photospheric temperature evolution $\left(643 \pm 60 \mathrm{R}_{\odot}\right)$ and $g$-band rise time - radius relation from $\operatorname{SNEC~}\left(619 \pm 10 \mathrm{R}_{\odot}\right)$. The values above are consistent with a core-collapse scenario from a typical RSG having an initial mass of $9.5-10 \mathrm{M}_{\odot}$.

\section{Acknowledgments}

We thank the support of the staffs at Xinglong Station (National Astronomical Observatory of China), Li-Jiang Observatory (Yunnan Astronomical Observatory of China), and Lick Observatory for assistance with the observations. We thank Melina Bersten for discussion on early detection of shock-breakout. We also acknowledge the use of public data from the Swift and Las Cumbres Observatory Global Telescope Network data archives. Some of the data presented herein were obtained at the W. M. Keck Observatory, which is operated as a scientific partnership among the California Institute of Technology, the 
University of California, and NASA; the observatory was made possible by the generous financial support of the W. M. Keck Foundation. KAIT and its ongoing operation were made possible by donations from Sun Microsystems, Inc., the Hewlett-Packard Company, AutoScope Corporation, Lick Observatory, the US National Science Foundation (NSF), the University of California, the Sylvia \& Jim Katzman Foundation, and the TABASGO Foundation. Research at Lick Observatory is partially supported by a generous gift from Google.

This work is supported by the Major State Basic Research Development Program (2013CB834903), the National Natural Science Foundation of China (NSFC grants 11178003, 11325313, and 11633002), and the Strategic Priority Research Program of Emergence of Cosmological Structures of the Chinese Academy of Sciences (grant No. XDB09000000). T.-M. Zhang is supported by the NSFC (grants 11203034). J.-J. Zhang is supported by the NSFC (grants 11403096), the Key Research Program of the CAS (Grant NO. KJZD-EW-M06) and the CAS "Light of West China" Program. DAH, CM, and GH are supported by NSF grant 1313484. A.V.F.'s group at U.C. Berkeley is grateful for financial assistance from NSF grant AST-1211916, the TABASGO Foundation, Gary and Cynthia Bengier, and the Christopher R. Redlich Fund. The work of A.V.F. was completed at the Aspen Center for Physics, which is supported by NSF grant PHY-1066293; he thanks the Center for its hospitality during the black holes workshop in June and July 2016. 


\section{REFERENCES}

Ahn, C. P., Alexandroff, R., Allende Prieto, C., et al. 2012, ApJS, 203, 21

Anderson, J. P., González-Gaitán, S., Hamuy, M., et al. 2014, ApJ, 786, 67

Andrews, J., Smith, N., Fong, W.-f., \& Milne, P. 2015, The Astronomer's Telegram, 7084, 1

Andrews, J. E., Krafton, K. M., Clayton, G. C., et al. 2016, MNRAS, 457, 3241

Arcavi, I., Gal-Yam, A., Cenko, S. B., et al. 2012, ApJ, 756, L30

Arnett, D. 1996, Supernovae and Nucleosynthesis: An Investigation of the History of Matter, from the Big Bang to the Present, by D. Arnett. Princeton: Princeton University Press, 1996.

Barbon, R., Ciatti, F., \& Rosino, L. 1979, A\&A, 72, 287

Bose, S., Valenti, S., Misra, K., et al. 2015, MNRAS, 450, 2373

Breeveld, A. A., Landsman, W., Holland, S. T., et al. 2011, American Institute of Physics Conference Series, 1358, 373

Brown, P. J., Holland, S. T., Immler, S., et al. 2009, AJ, 137, 4517

Brown, T. M., Baliber, N., Bianco, F. B., et al. 2013, PASP, 125, 1031

Brown, P. J., Breeveld, A. A., Holland, S., Kuin, P., \& Pritchard, T. 2014, Ap\&SS, 354, 89

Cardelli, J. A., Clayton, G. C., \& Mathis, J. S. 1989, ApJ, 345, 245

Dall'Ora, M., Botticella, M. T., Pumo, M. L., et al. 2014, ApJ, 787, 139

Dessart, L., \& Hillier, D. J. 2005, A\&A, 439, 671

Dessart, L., Livne, E., \& Waldman, R. 2010, MNRAS, 408, 827 
Dhungana, G., Kehoe, R., Vinko, J., et al. 2016, ApJ, 822, 6

Elias-Rosa, N., Tartaglia, L., Cappellaro, E., et al. 2014, The Astronomer's Telegram, 6440, 1

Elmhamdi, A., Danziger, I. J., Chugai, N., et al. 2003, MNRAS, 338, 939

Falk, S. W., \& Arnett, W. D. 1977, ApJS, 33, 515

Faran, T., Poznanski, D., Filippenko, A. V., et al. 2014, MNRAS, 442, 844

Faran, T., Poznanski, D., Filippenko, A. V., et al. 2014, MNRAS, 445, 554

Filippenko, A. V. 1997, ARA\&A, 35, 309

Filippenko, A. V., Li, W. D., Treffers, R. R., \& Modjaz, M. 2001, IAU Colloq. 183: Small Telescope Astronomy on Global Scales, 246, 121

Ganeshalingam, M., Li, W., Filippenko, A. V., et al. 2010, ApJS, 190, 418

Garnavich, P. M., Tucker, B. E., Rest, A., et al. 2016, ApJ, 820, 23

Gezari, S., Jones, D. O., Sanders, N. E., et al. 2015, ApJ, 804, 28

González-Gaitán, S., Tominaga, N., Molina, J., et al. 2015, MNRAS, 451, 2212

Hamuy, M. 2003, ApJ, 582, 905

Heger, A., Fryer, C. L., Woosley, S. E., Langer, N., \& Hartmann, D. H. 2003, ApJ, 591, 288

Holoien, T. W.-S., Prieto, J. L., Kochanek, C. S., et al. 2014, The Astronomer's Telegram, 6436,1

Horne, K. 1986, PASP, 98, 609 
Huang, F., Li, J.-Z., Wang, X.-F., et al. 2012, Research in Astronomy and Astrophysics, 12, 1585

Huang, F., Wang, X., Zhang, J., et al. 2015, ApJ, 807, 59

Inserra, C., Turatto, M., Pastorello, A., et al. 2011, MNRAS, 417, 261

Inserra, C., Turatto, M., Pastorello, A., et al. 2012, MNRAS, 422, 1122

Inserra, C., Pastorello, A., Turatto, M., et al. 2013, A\&A, 555, A142

Kasen, D., \& Woosley, S. E. 2009, ApJ, 703, 2205

Klein, R. I., \& Chevalier, R. A. 1978, ApJ, 223, L109

Leonard, D. C., Filippenko, A. V., Gates, E. L., et al. 2002, PASP, 114, 35

Li, W., Wang, X., Van Dyk, S. D., et al. 2007, ApJ, 661, 1013

Li, W., Leaman, J., Chornock, R., et al. 2011, MNRAS, 412, 1441

Maguire, K., Di Carlo, E., Smartt, S. J., et al. 2010, MNRAS, 404, 981

Monet, D. 1998, USNO-A2.0, by Monet, David. [Flagstaff, AZ] : U.S. Naval Observatory, c1998. . United States Naval Observatory

Morozova, V., Piro, A. L., Renzo, M., et al. 2015, ApJ, 814, 63

Morozova, V., Piro, A. L., Renzo, M., \& Ott, C. D. 2016, arXiv:1603.08530

Morrell, N., Marion, G. H., Kirshner, R. P., Hsiao, E. Y., \& Stritzinger, M. 2014, The Astronomer's Telegram, 6442, 1

Nadyozhin, D. K. 2003, MNRAS, 346, 97

Nakano, S., Itagaki, K., Yusa, T., et al. 2014, Central Bureau Electronic Telegrams, 3963, 1 
Olivares E., F., Hamuy, M., Pignata, G., et al. 2010, ApJ, 715, 833

Pastorello, A., Valenti, S., Zampieri, L., et al. 2009, MNRAS, 394, 2266

Pastorello, A., Pumo, M. L., Navasardyan, H., et al. 2012, A\&A, 537, A141

Patat, F., Barbon, R., Cappellaro, E., \& Turatto, M. 1994, A\&A, 282, 731

Pumo, M. L., Zampieri, L., \& Turatto, M. 2010, Memorie della Societa Astronomica Italiana Supplementi, 14, 123

Pumo, M. L., \& Zampieri, L. 2011, ApJ, 741, 41

Pumo, M. L., \& Zampieri, L. 2013, MNRAS, 434, 3445

Quimby, R. M., Wheeler, J. C., Höflich, P., et al. 2007, ApJ, 666, 1093

Rabinak, I., \& Waxman, E. 2011, ApJ, 728, 63

Roming, P. W. A., Kennedy, T. E., Mason, K. O., et al. 2005, Space Sci. Rev., 120, 95

Rubin, A., Gal-Yam, A., De Cia, A., et al. 2016, ApJ, 820, 33

Sahu, D. K., Anupama, G. C., Srividya, S., \& Muneer, S. 2006, MNRAS, 372, 1315

Sanders, N. E., Soderberg, A. M., Gezari, S., et al. 2015, ApJ, 799, 208

Schlafly, E. F., \& Finkbeiner, D. P. 2011, ApJ, 737, 103

Schawinski, K., Justham, S., Wolf, C., et al. 2008, Science, 321, 223

Smartt, S. J., Eldridge, J. J., Crockett, R. M., \& Maund, J. R. 2009, MNRAS, 395, 1409

Smartt, S. J. 2009, ARA\&A, 47, 63

Sorce, J. G., Tully, R. B., Courtois, H. M., et al. 2014, MNRAS, 444, 527 
Tomasella, L., Cappellaro, E., Fraser, M., et al. 2013, MNRAS, 434, 1636

Tominaga, N., Blinnikov, S., Baklanov, P., et al. 2009, ApJ, 705, L10

Valenti, S., Sand, D., Pastorello, A., et al. 2014, MNRAS, 438, L101

Valenti, S., Howell, D. A., Stritzinger, M. D., et al. 2016, MNRAS, 459, 3939

Wang, X., Li, W., Filippenko, A. V., et al. 2008, ApJ, 675, 626

Zampieri, L., Pastorello, A., Turatto, M., et al. 2003, MNRAS, 338, 711 


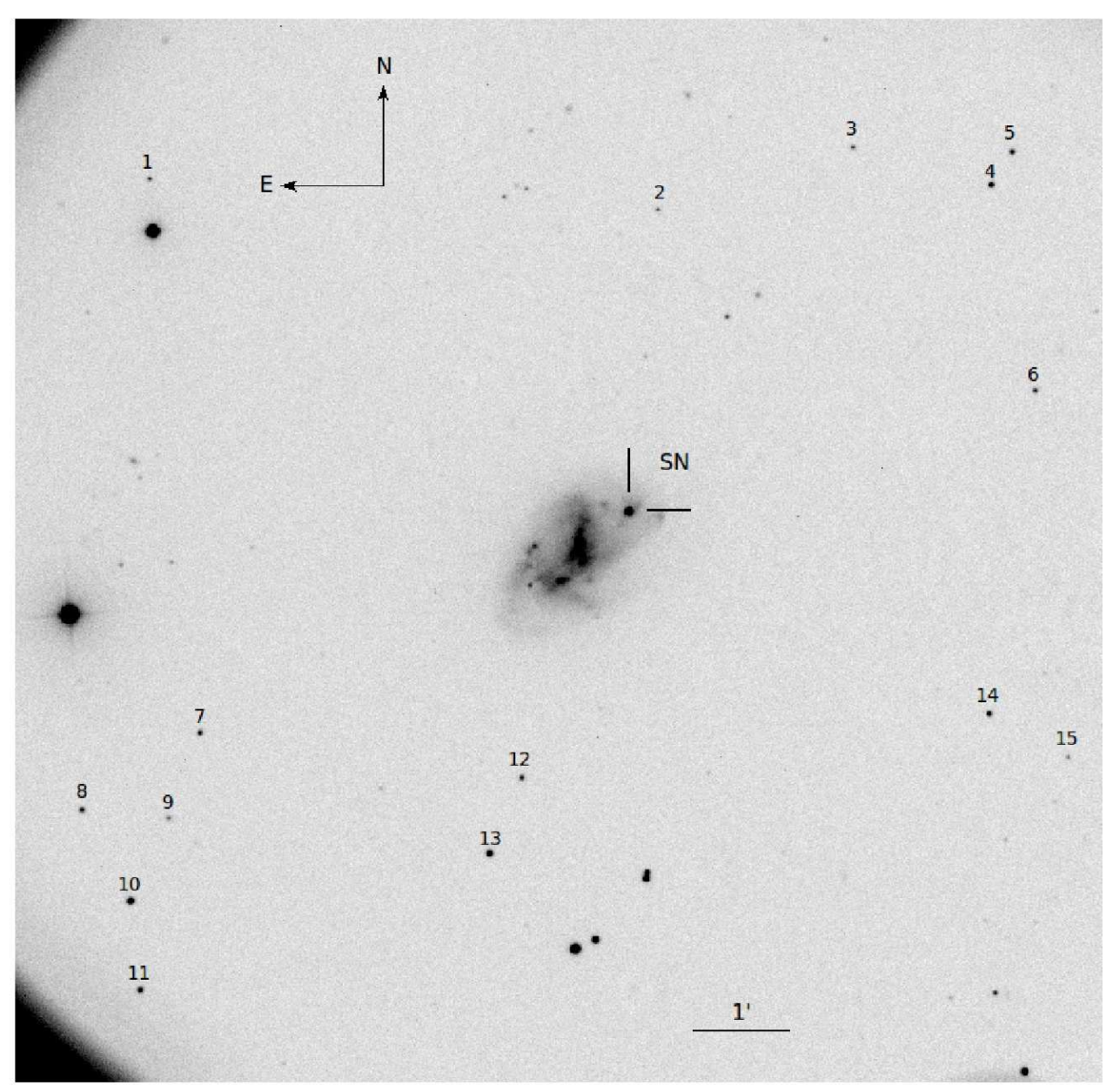

Fig. 1.- SN 2014cx in NGC 337. This $R$-band image was taken on 2014 Oct. 25 with the $80 \mathrm{~cm}$ Tsinghua-NAOC telescope. SN 2014cx and the 15 local sequence stars are marked. 


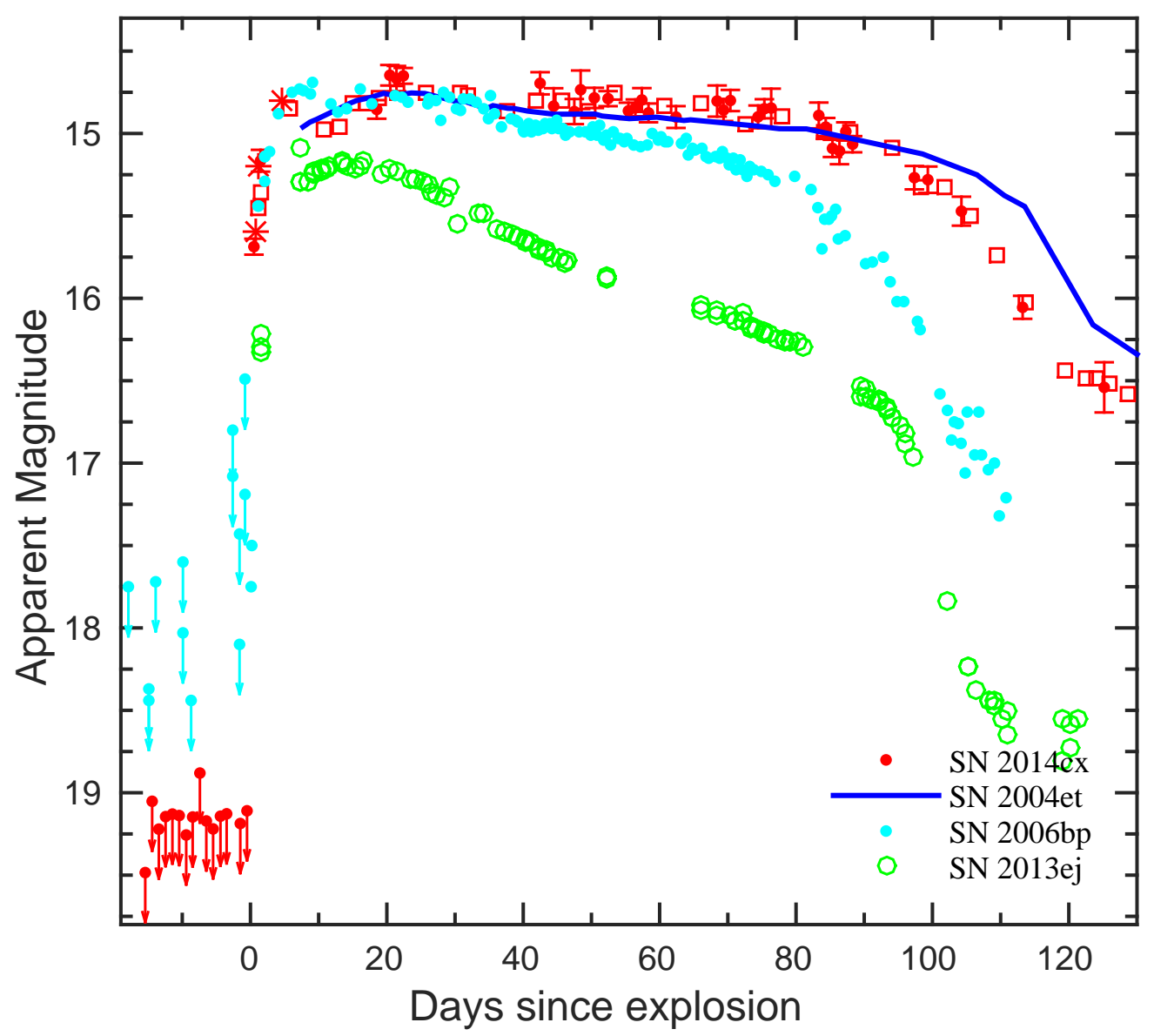

Fig. 2.- KAIT unfiltered (red circle) and LCOGT $r$ band (red square) light curve of SN 2014cx unfiltered detections from the Bright Supernovae website (http://www.rochesterastronomy.org/snimages/) (red star), SN 2004et in the $R$ band (blue line), SN 2006bp (cyan), and SN 2013ej (green). For KAIT data, detections are plotted with filled circles, and arrows represent $4 \sigma$ upper limits. 


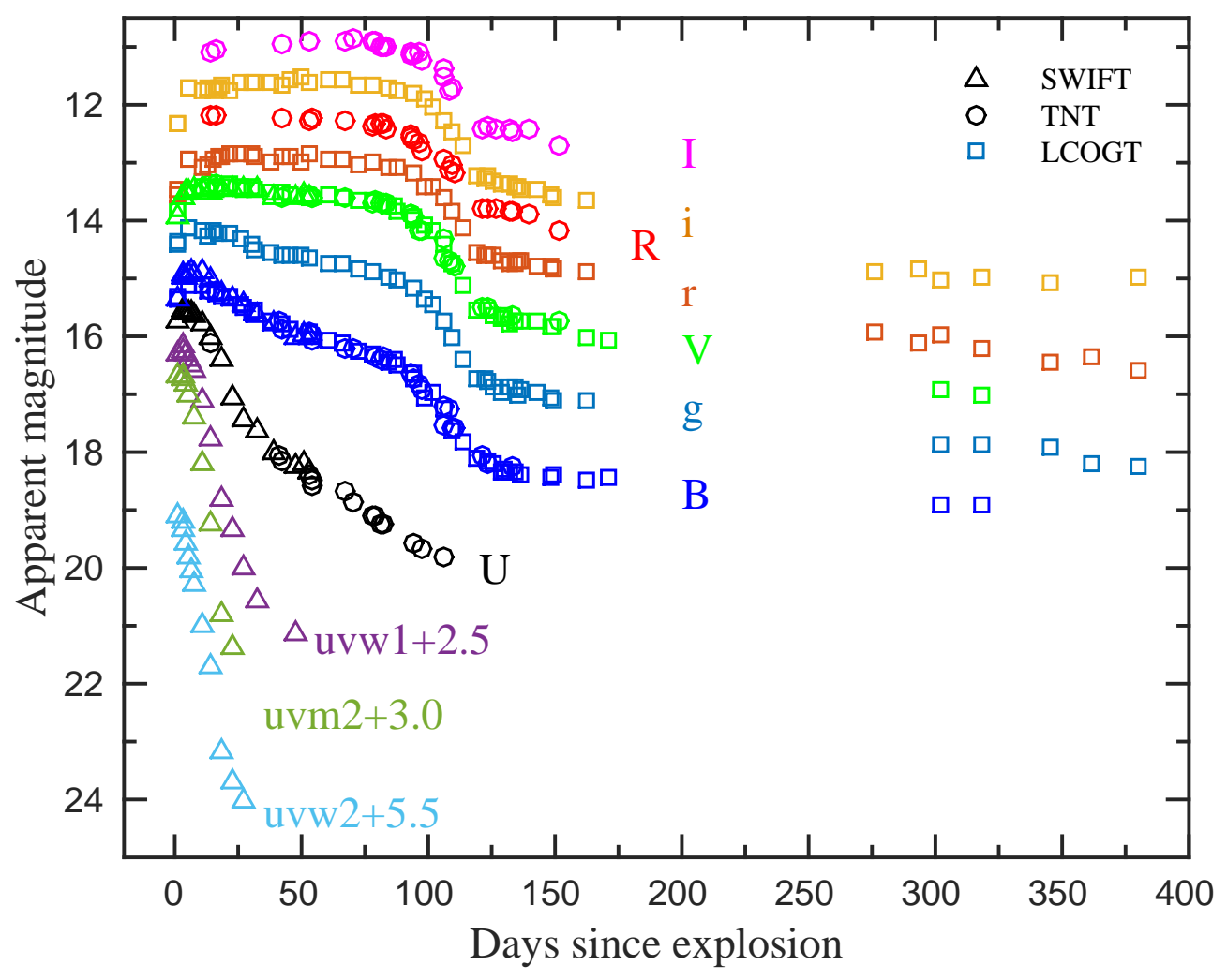

Fig. 3.- The photometric evolution of SN 2014cx in UV and optical bands. The phase is given relative to the estimated explosion date, $\mathrm{MJD}=56,901.89$. 


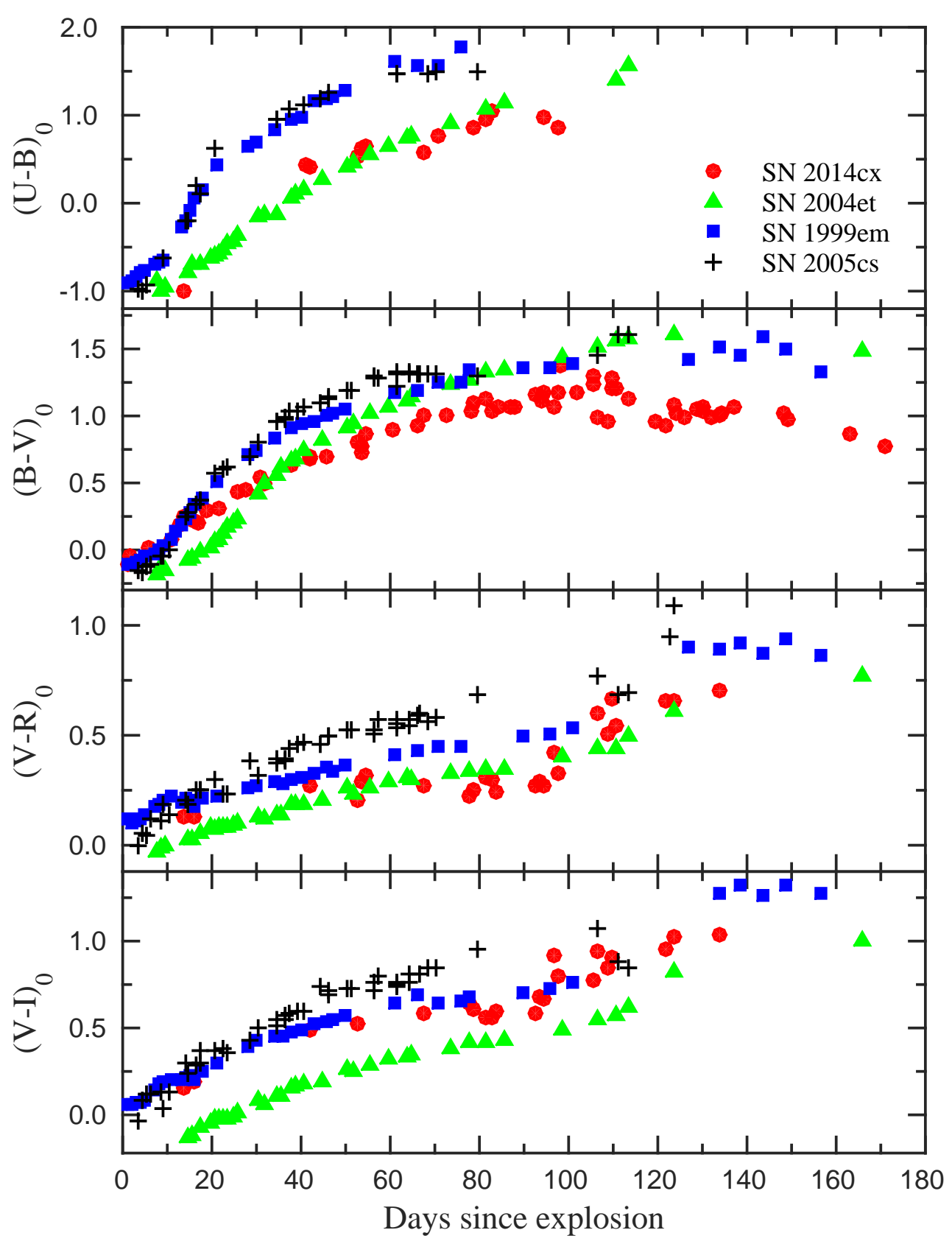

Fig. 4.- The dereddening optical color evolution of SN 2014cx compared with those of other SNe IIP : SNe 1999em, 2004et, and 2005cs. 


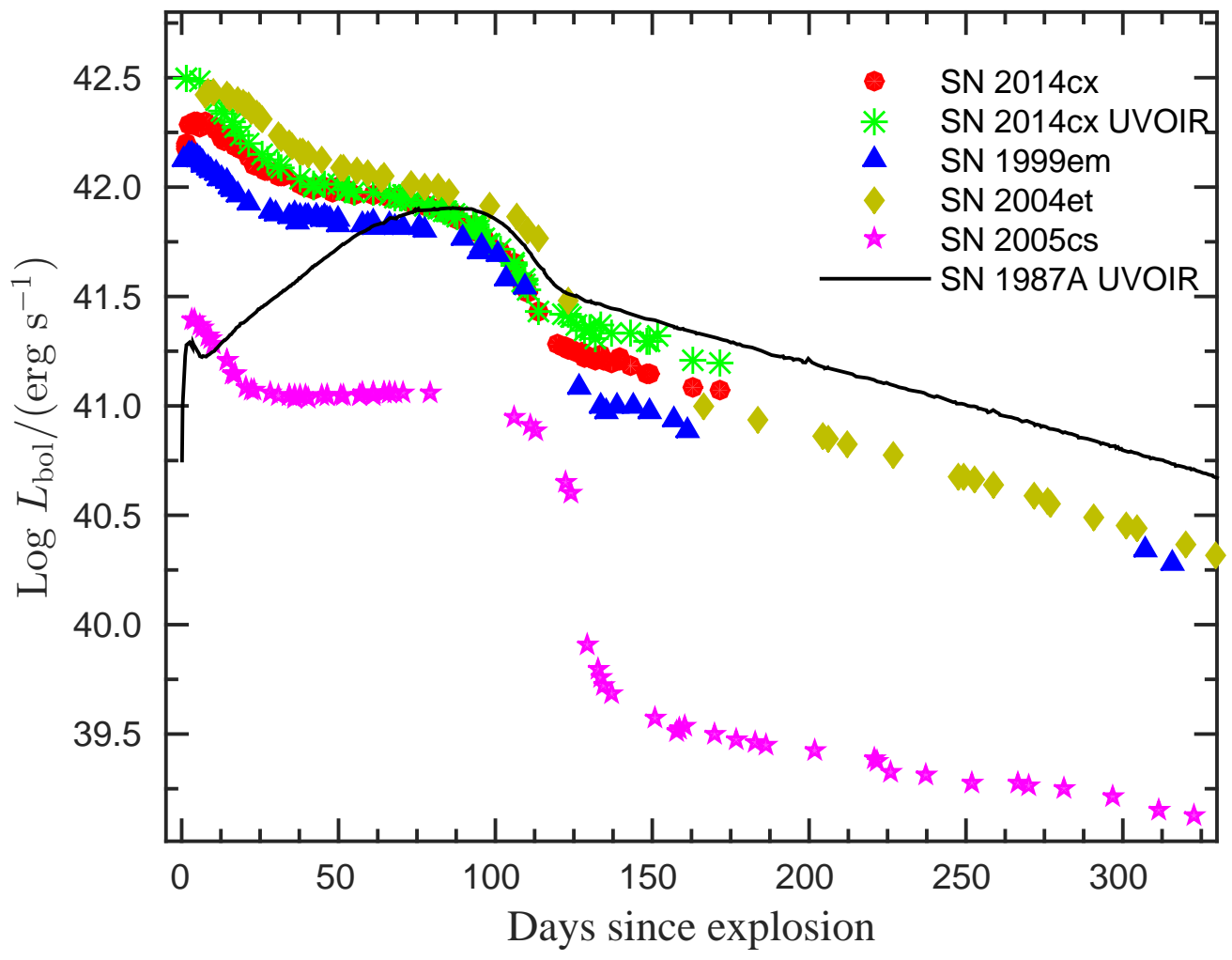

Fig. 5.- Comparison of the bolometric luminosity evolution of SN 2014cx and other wellstudied SNe IIP. 


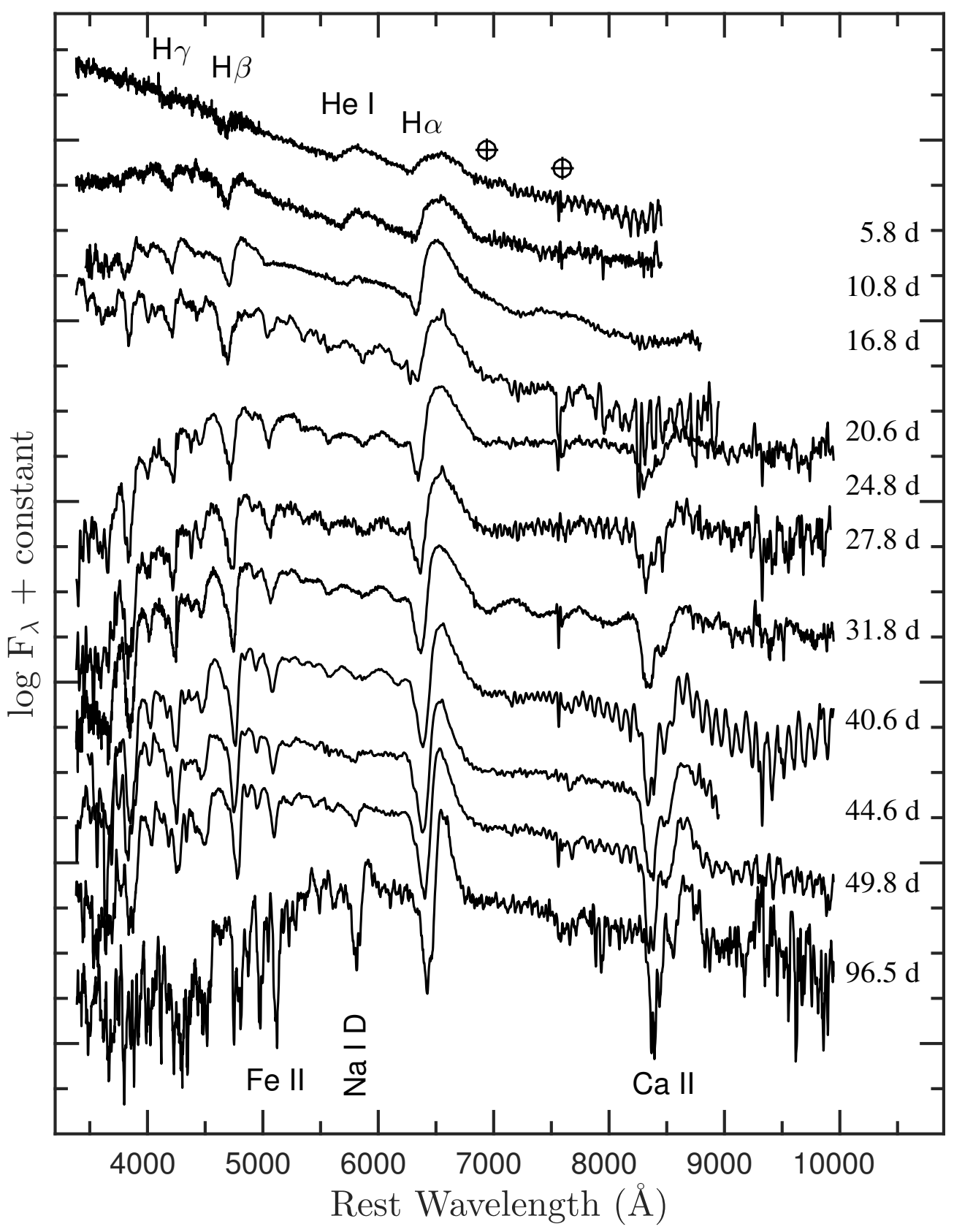

Fig. 6.- Spectroscopic evolution of SN 2014cx during the photospheric phase. The spectra have been corrected for the redshift of the host galaxy NGC 337 ( $z=0.00549$, via NED). Some key features are labelled, as are residuals from telluric absorption lines. The oscillations in some of the near-infrared spectra (most notably, at $40.6 \mathrm{~d}$ ) are caused by incomplete removal of CCD fringing. 


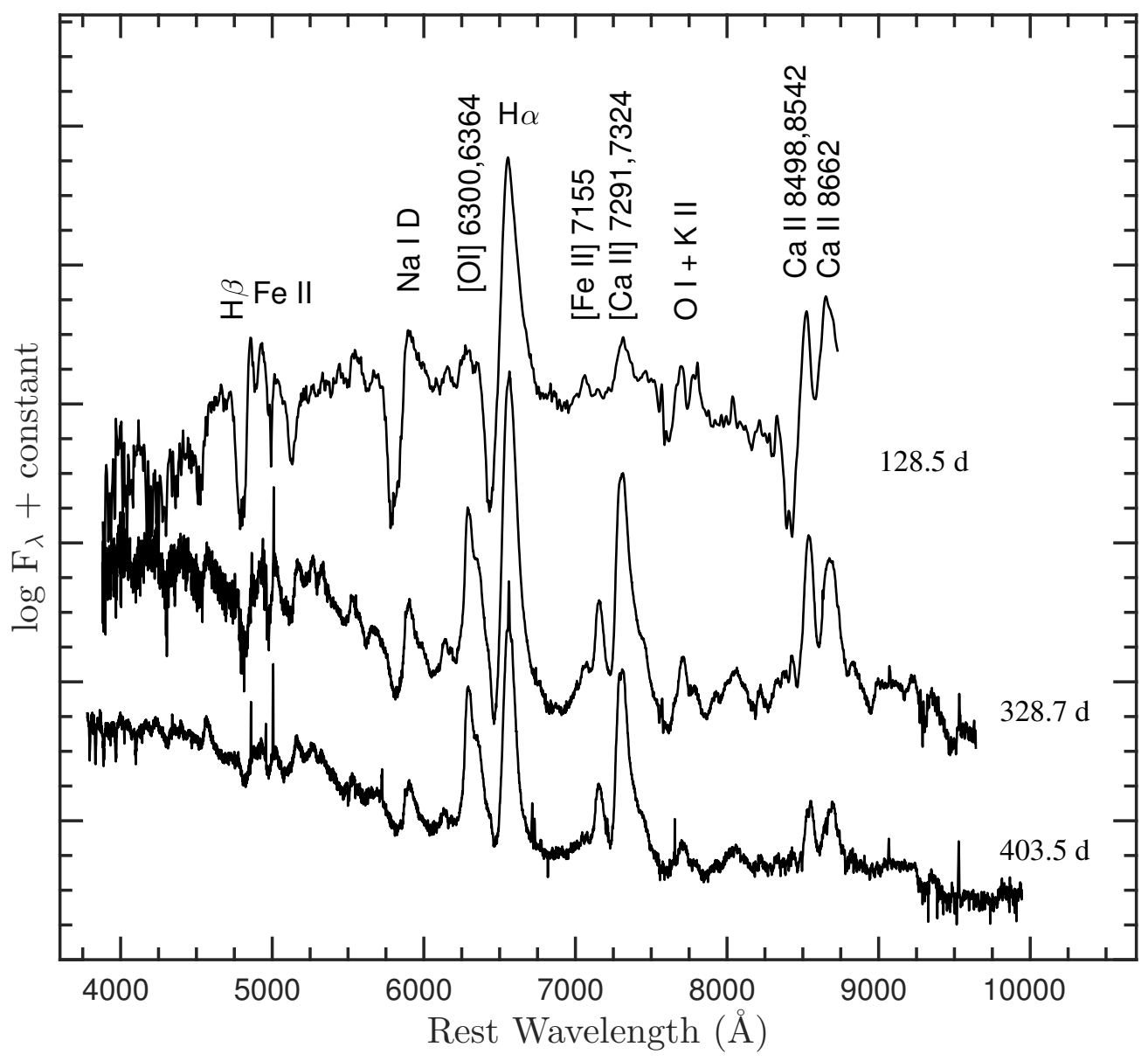

Fig. 7.- Spectroscopic evolution of SN 2014cx during the nebular phase, when emission lines are dominant features. 


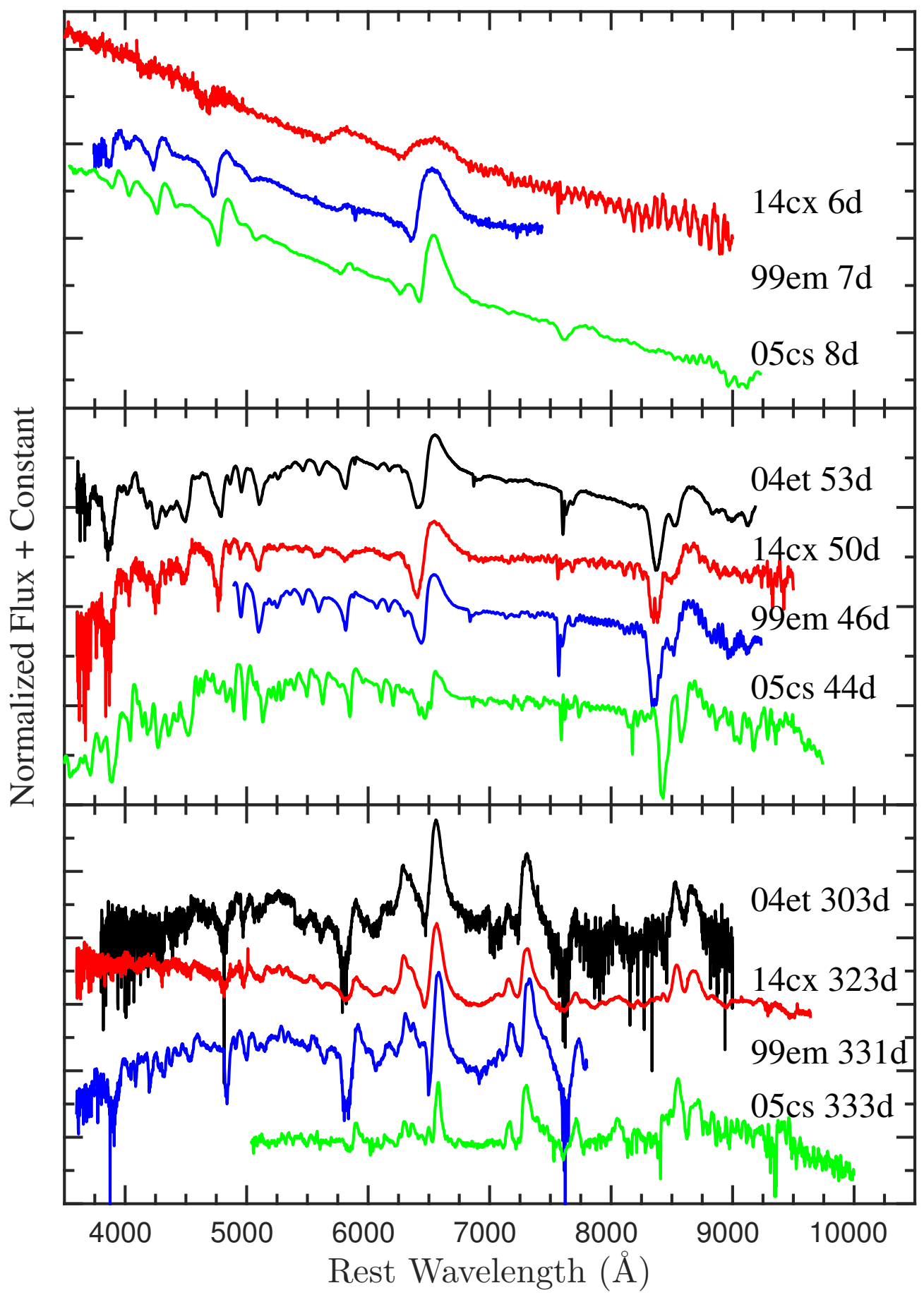

Fig. 8. - Comparison of the reddening- and Doppler-corrected spectra of SNe 2014cx, 2004et, $1999 \mathrm{em}$, and 2005cs at similar phases. Top panel, one week after explosion; middle panel, about 50 days after explosion; bottom panel, about one year after explosion. 


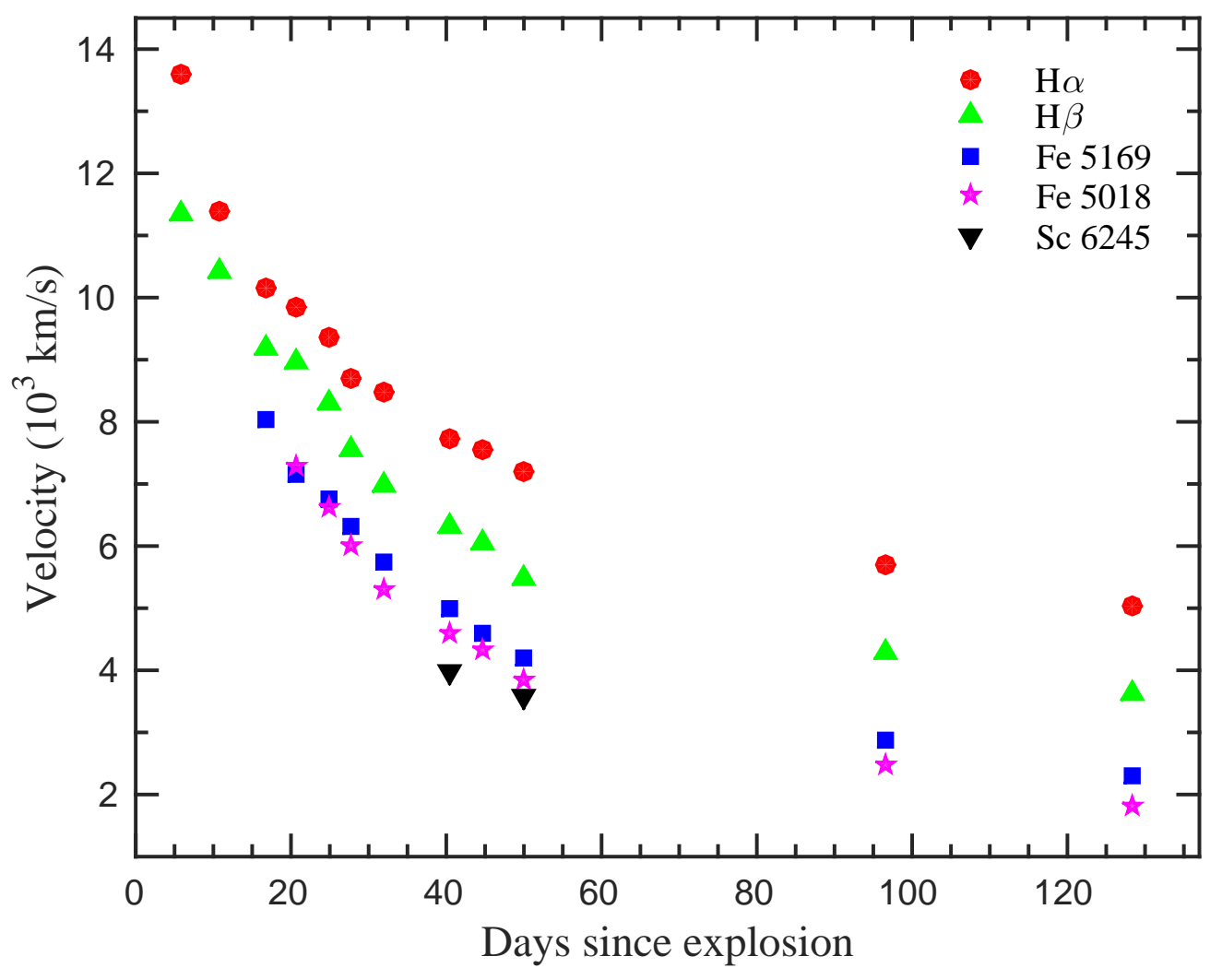

Fig. 9.- The velocity evolution of $\mathrm{H} \alpha, \mathrm{H} \beta$, and Fe II. The velocities are estimated using the Doppler shift of the absorption minima. 


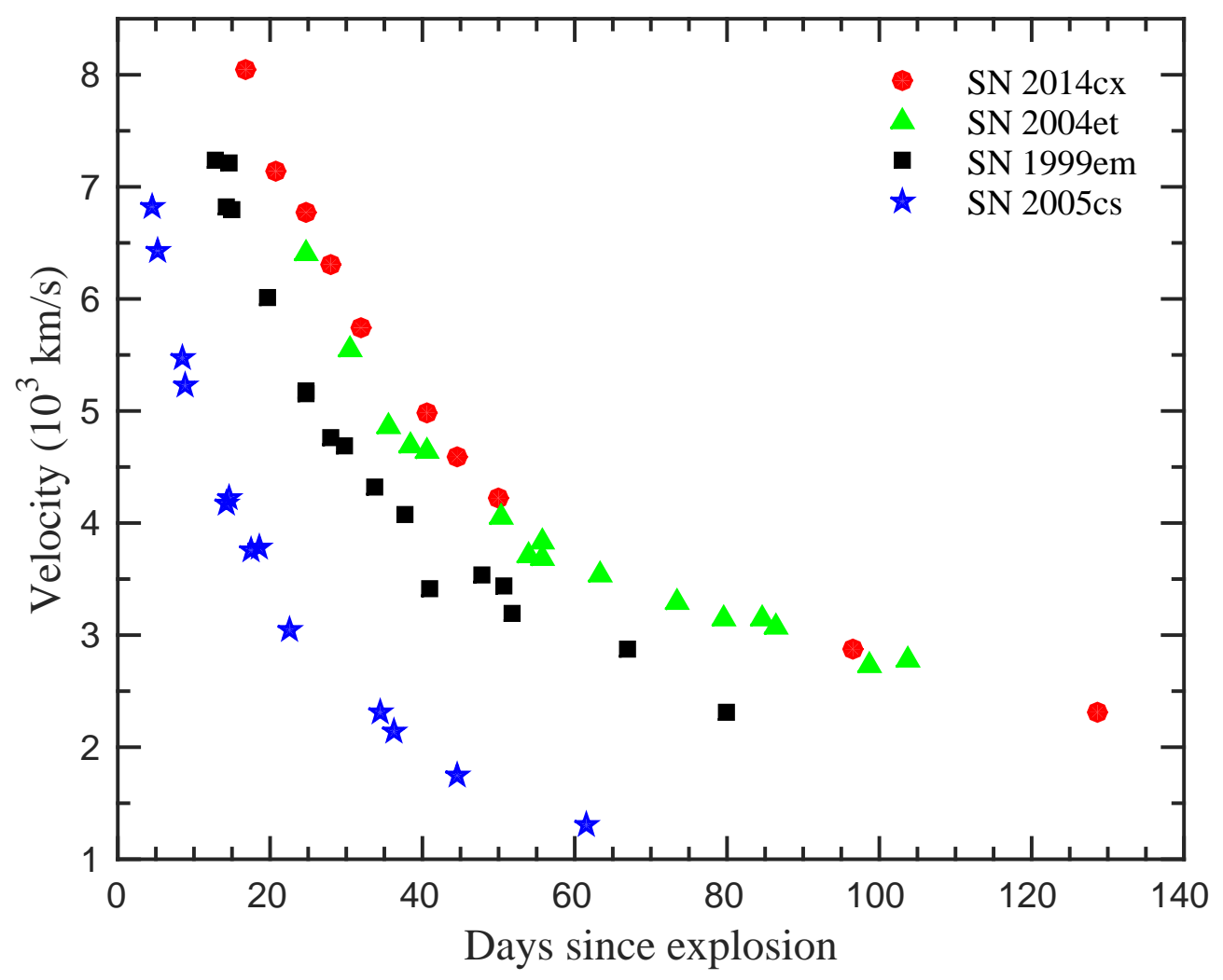

Fig. 10.- Comparison of the expansion velocities of SN 2014cx measured from Fe II $\lambda 5169$ to those of the luminous Type IIP SN 2004et, the normal SN 1999em, and the subluminous SN 2005cs. 


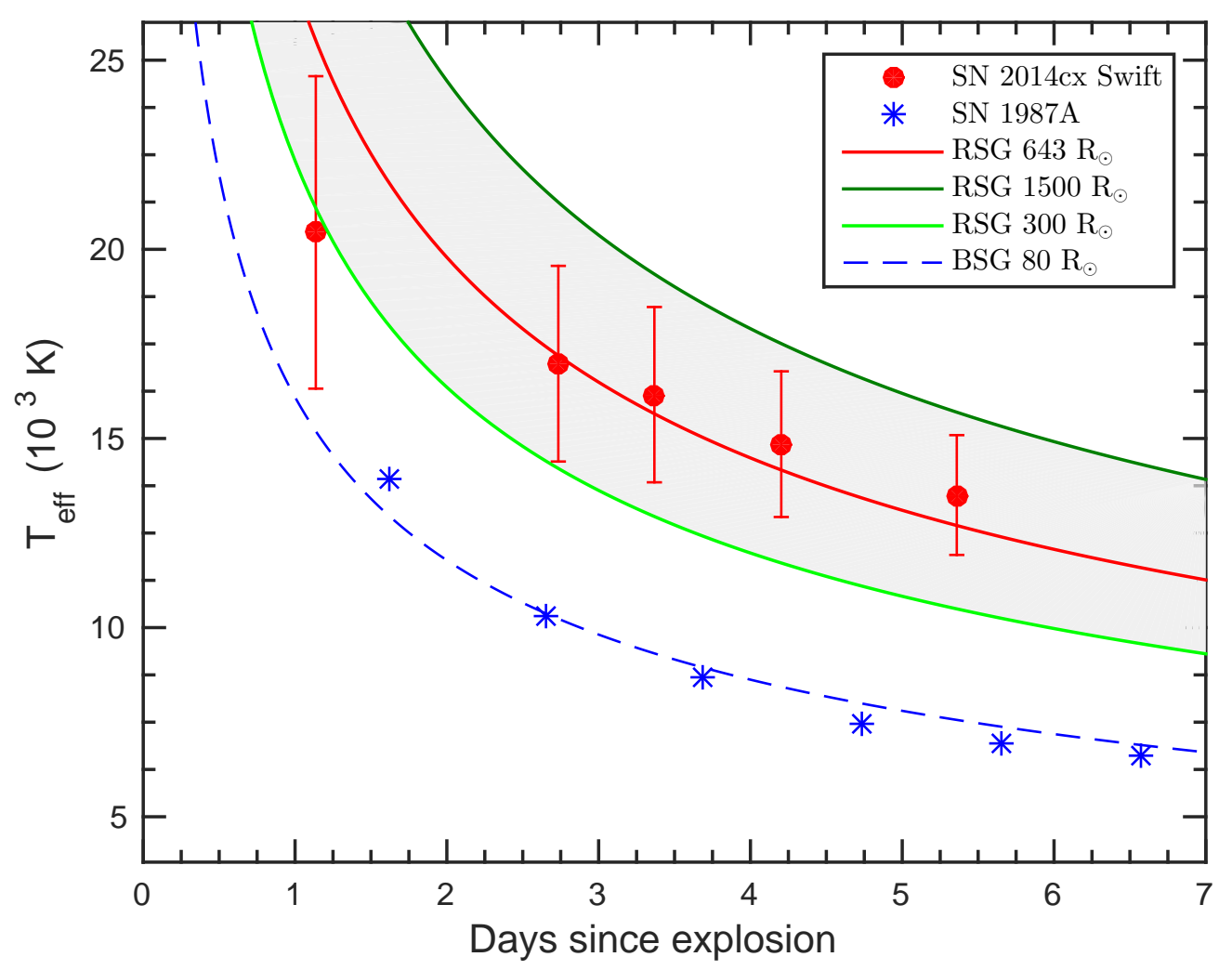

Fig. 11.- Constraining the progenitor radius using the Rabinak \& Waxman (2011) prescription. The red solid line is the best fit for a RSG of $643 \mathrm{R}_{\odot}$, and the blue dashed line is for a $\mathrm{BSG}$ of $80 \mathrm{R}_{\odot}$ for $\mathrm{SN} 1987 \mathrm{~A}$. 

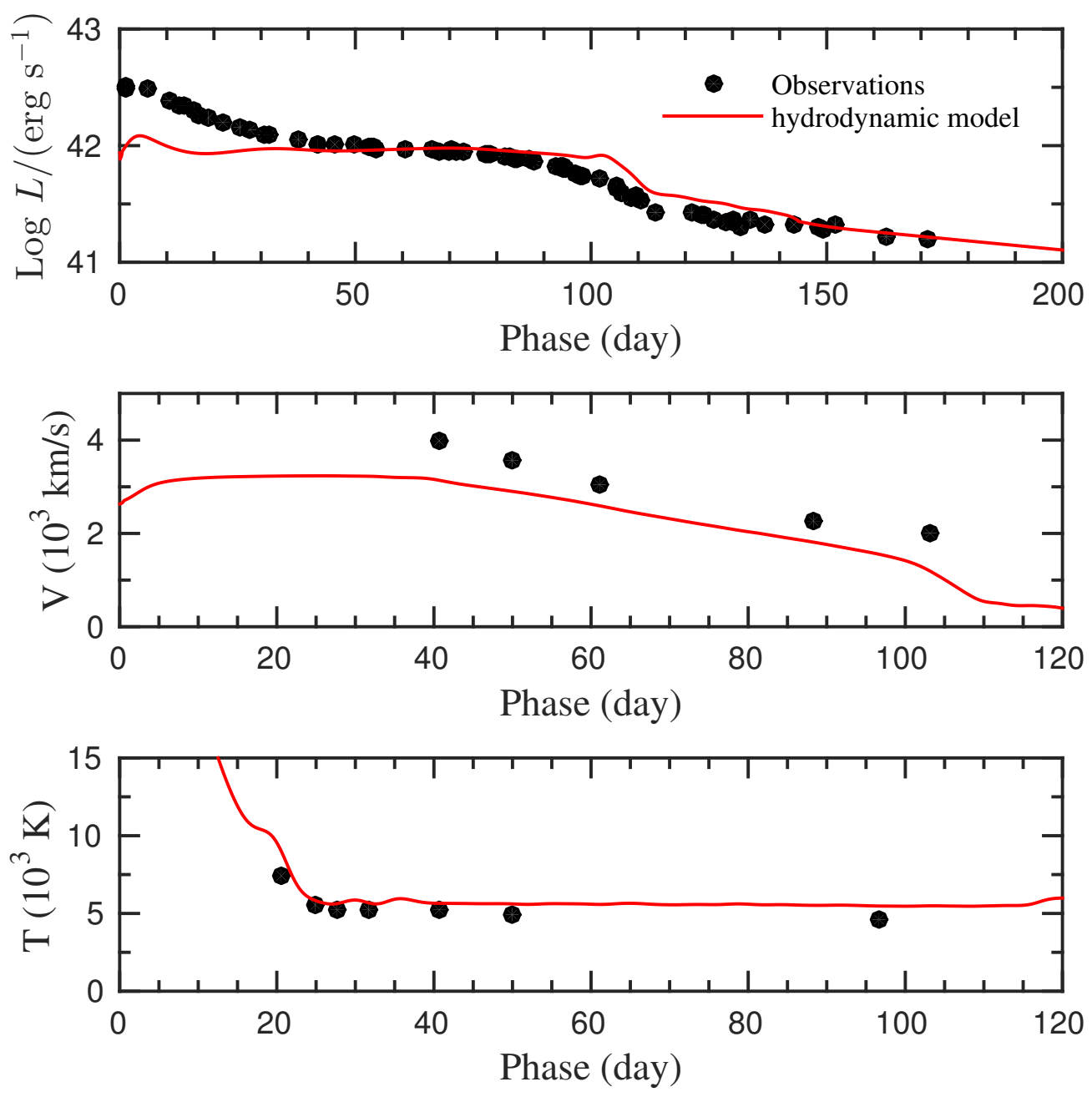

Fig. 12.- Comparison of the evolution of the main observables of SN 2014cx with the bestfit model computed with the general-relativistic, radiation-hydrodynamics code (total energy $\sim 0.4 \times 10^{51} \mathrm{erg}$, initial radius $4 \times 10^{13} \mathrm{~cm}$, envelope mass $\left.8 \mathrm{M}_{\odot}\right)$. Top, middle, and bottom panels show the bolometric light curve, the photospheric velocity, and the photospheric temperature as a function of time, respectively. To estimate the photospheric velocity from observations, we used the value inferred from the Fe II $\lambda 5169$ line. 
Table 1: Photometric Standard Stars in the Field of SN 2014cx (1 $\sigma$ Uncertainties).

\begin{tabular}{cccccccc}
\hline \hline Star & $\alpha_{\mathrm{J} 2000}$ & $\delta_{\mathrm{J} 2000}$ & $U$ & $B$ & $V$ & $R$ & $I$ \\
ID & $(\mathrm{h} \mathrm{m} \mathrm{s})$ & $\left({ }^{\prime \prime \prime}\right)$ & $(\mathrm{mag})$ & $(\mathrm{mag})$ & $(\mathrm{mag})$ & $(\mathrm{mag})$ & $(\mathrm{mag})$ \\
\hline 1 & $1: 00: 07.08$ & $-7: 30: 33.54$ & $20.07(09)$ & $19.35(06)$ & $18.31(04)$ & $17.69(05)$ & $17.06(09)$ \\
2 & $1: 00: 11.55$ & $-7: 37: 03.82$ & $18.61(04)$ & $18.39(05)$ & $17.56(03)$ & $17.08(04)$ & $16.59(07)$ \\
3 & $0: 59: 45.87$ & $-7: 31: 12.11$ & $20.17(11)$ & $19.71(06)$ & $18.66(04)$ & $18.04(04)$ & $17.43(08)$ \\
4 & $0: 59: 37.53$ & $-7: 30: 40.79$ & $21.32(25)$ & $20.43(08)$ & $18.79(05)$ & $17.69(11)$ & $16.26(22)$ \\
5 & $0: 59: 30.12$ & $-7: 37: 08.94$ & $18.66(10)$ & $18.56(03)$ & $18.20(02)$ & $17.98(03)$ & $17.77(05)$ \\
6 & $0: 59: 53.05$ & $-7: 37: 00.70$ & $18.26(04)$ & $18.24(04)$ & $17.52(03)$ & $17.08(03)$ & $16.65(06)$ \\
7 & $1: 00: 09.58$ & $-7: 38: 58.23$ & $18.38(04)$ & $18.07(05)$ & $17.19(03)$ & $16.66(04)$ & $16.12(08)$ \\
8 & $1: 00: 06.41$ & $-7: 36: 20.14$ & $20.47(15)$ & $19.52(08)$ & $17.96(04)$ & $17.01(07)$ & $16.03(15)$ \\
9 & $0: 59: 30.89$ & $-7: 30: 49.81$ & $19.23(06)$ & $18.56(05)$ & $17.58(04)$ & $17.00(04)$ & $16.44(08)$ \\
10 & $1: 00: 07.93$ & $-7: 37: 12.15$ & $19.69(08)$ & $19.38(05)$ & $18.45(03)$ & $17.89(04)$ & $17.31(08)$ \\
11 & $0: 59: 54.59$ & $-7: 37: 46.63$ & $18.04(04)$ & $17.44(05)$ & $16.47(03)$ & $15.89(04)$ & $15.34(08)$ \\
12 & $1: 00: 09.75$ & $-7: 38: 02.37$ & $17.27(03)$ & $17.11(05)$ & $16.28(03)$ & $15.77(04)$ & $15.24(08)$ \\
13 & $0: 59: 31.84$ & $-7: 31: 09.63$ & $20.16(10)$ & $19.10(08)$ & $17.56(05)$ & $16.62(07)$ & $15.61(15)$ \\
14 & $0: 59: 33.31$ & $-7: 36: 38.75$ & $18.08(09)$ & $17.86(04)$ & $17.12(03)$ & $16.69(03)$ & $16.28(06)$ \\
\hline & $0: 59: 30.53$ & $-7: 33: 19.29$ & $16.08(03)$ & $17.22(04)$ & $17.29(03)$ & $17.37(04)$ & $17.50(08)$ \\
\hline
\end{tabular}


Table 2:: Optical Photometry of SN 2014cx from TNT (1 $\sigma$ Uncertainties).

\begin{tabular}{|c|c|c|c|c|c|c|c|}
\hline $\begin{array}{c}\text { UT Date } \\
(\mathrm{yy} / \mathrm{mm} / \mathrm{dd})\end{array}$ & MJD & $\begin{array}{c}\text { Phase }^{a} \\
\text { (day) }\end{array}$ & $\begin{array}{c}U \\
(\mathrm{mag})\end{array}$ & $\begin{array}{c}B \\
(\mathrm{mag})\end{array}$ & $\begin{array}{c}V \\
(\mathrm{mag})\end{array}$ & $\begin{array}{c}R \\
(\mathrm{mag})\end{array}$ & $\begin{array}{c}I \\
(\mathrm{mag})\end{array}$ \\
\hline 2014 Sep. 15 & 56915.75 & 13.86 & $14.30(04)$ & $15.23(03)$ & $14.89(04)$ & $14.70(10)$ & $14.60(05)$ \\
\hline 2014 Sep. 17 & 56917.75 & 15.86 & $\cdots$ & $15.14(03)$ & $14.89(07)$ & $14.70(07)$ & $14.56(03)$ \\
\hline 2014 Oct. 12 & 56942.75 & 40.86 & $16.26(08$ & $15.75(01)$ & & . & \\
\hline 2014 Oct. 13 & 56943.75 & 41.86 & $16.37(12)$ & $15.87(04)$ & $15.08(04)$ & $14.75(06)$ & $14.46(04)$ \\
\hline 2014 Oct. 21 & 56951.50 & 49.61 & $16.49(06)$ & $15.87(04)$ & $15.01(03)$ & $14.67(04)$ & $14.40(04)$ \\
\hline 2014 Oct. 24 & 56954.50 & 52.61 & $16.57(11)$ & $15.95(03)$ & $15.05(02)$ & $14.78(04)$ & $14.39(04)$ \\
\hline 2014 Oct. 25 & 56955.50 & 53.61 & $16.67(06)$ & $15.98(04)$ & $15.11(02)$ & $14.75(07)$ & \\
\hline 2014 Oct. 26 & 56956.50 & 54.61 & $16.79(05)$ & $16.07(02)$ & $15.11(03)$ & $14.73(05)$ & \\
\hline 2014 Nov. 08 & 56969.50 & 67.61 & $16.87(09)$ & $16.27(03)$ & $15.11(03)$ & $14.77(03)$ & $14.38(04)$ \\
\hline 2014 Nov. 10 & 56971.50 & 69.61 & $17.07(08)$ & $16.27(05)$ & $15.03(03)$ & $14.75(04)$ & $14.44(05)$ \\
\hline 2014 Nov. 11 & 56972.50 & 70.61 & $17.07(05)$ & $16.23(03)$ & $15.06(04)$ & $14.74(04)$ & $14.38(04)$ \\
\hline 2014 Nov. 12 & 56973.50 & 71.61 & & & $15.07(03)$ & $14.78(02)$ & \\
\hline 2014 Nov. 18 & 56979.50 & 77.61 & $17.28(16)$ & & $15.18(05)$ & $14.89(07)$ & $14.40(06)$ \\
\hline 2014 Nov. 19 & 56980.50 & 78.61 & $17.30(15)$ & $16.36(03)$ & $15.15(04)$ & $14.84(05)$ & $14.41(05)$ \\
\hline 2014 Nov. 22 & 56983.50 & 81.61 & $17.46(06)$ & $16.42(05)$ & $15.19(05)$ & $14.82(06)$ & $14.50(04)$ \\
\hline 2014 Nov. 23 & 56984.50 & 82.61 & $17.47(11)$ & $16.34(02)$ & $15.20(04)$ & $14.84(05)$ & $14.51(03)$ \\
\hline 2014 Nov. 24 & 56985.50 & 83.61 & & & $15.22(02)$ & $14.91(03)$ & $14.48(03)$ \\
\hline 2014 Dec. 03 & 56994.50 & 92.61 & $\cdots$ & $16.62(04)$ & $15.37(02)$ & $15.03(05)$ & $14.64(02)$ \\
\hline 2014 Dec. 04 & 56995.50 & 93.61 & $\cdots$ & $\cdots$ & $15.41(02)$ & $15.06(03)$ & $14.59(02)$ \\
\hline 2014 Dec. 05 & 56996.50 & 94.61 & $17.79(25)$ & $16.73(04)$ & $15.45(02)$ & $15.12(03)$ & $14.65(06)$ \\
\hline 2014 Dec. 07 & 56998.50 & 96.61 & $\cdots$ & $16.82(05)$ & $15.66(02)$ & $15.18(02)$ & $14.61(04)$ \\
\hline
\end{tabular}




\begin{tabular}{llllllll} 
2014 Dec. 08 & 56999.50 & 97.61 & $17.88(11)$ & $16.94(04)$ & $15.67(06)$ & $15.28(07)$ & $14.74(06)$ \\
2014 Dec. 16 & 57007.50 & 105.61 & $\ldots$ & $17.22(05)$ & $15.82(03)$ & $99.50(05)$ & $14.90(03)$ \\
2014 Dec. 17 & 57008.50 & 106.61 & $18.03(09)$ & $17.52(06)$ & $16.13(04)$ & $15.46(06)$ & $15.04(04)$ \\
2014 Dec. 19 & 57010.50 & 108.61 & $\ldots$ & $17.27(05)$ & $16.21(04)$ & $15.64(05)$ & $15.23(05)$ \\
2014 Dec. 20 & 57011.50 & 109.61 & $\ldots$ & $17.57(07)$ & $16.10(06)$ & $15.51(05)$ & $15.22(07)$ \\
2014 Dec. 21 & 57012.50 & 110.61 & $\ldots$ & $17.58(05)$ & $16.28(03)$ & $15.68(04)$ & $\ldots$ \\
2015 Jan. 01 & 57023.50 & 121.61 & $\ldots$ & $18.04(09)$ & $17.01(06)$ & $16.30(03)$ & $15.92(04)$ \\
2015 Jan. 03 & 57025.50 & 123.61 & $\ldots$ & $18.20(06)$ & $17.02(07)$ & $16.31(05)$ & $15.86(03)$ \\
2015 Jan. 06 & 57028.50 & 126.61 & $\ldots$ & $\ldots$ & $\ldots$ & $16.31(05)$ & $15.91(05)$ \\
2015 Jan. 07 & 57029.50 & 127.61 & $\ldots$ & $\ldots$ & $17.01(04)$ & $16.39(07)$ & $15.89(06)$ \\
2015 Jan. 08 & 57030.50 & 128.61 & $\ldots$ & $18.09(05)$ & $17.21(05)$ & $16.37(05)$ & $15.91(05)$ \\
2015 Jan. 12 & 57034.50 & 132.61 & $\ldots$ & $\ldots$ & $\ldots$ & $16.34(02)$ & $15.93(04)$ \\
2015 Jan. 13 & 57035.50 & 133.61 & $\ldots$ & $18.23(05)$ & $17.13(04)$ & $16.36(04)$ & $15.95(04)$ \\
2015 Jan. 18 & 57040.50 & 138.61 & $\ldots$ & $\ldots$ & $\ldots$ & $16.42(03)$ & $16.00(02)$ \\
2015 Jan. 19 & 57041.50 & 139.61 & $\ldots$ & $\ldots$ & $17.18(04)$ & $16.41(05)$ & $16.01(05)$ \\
2015 Jan. 31 & 57053.50 & 151.61 & $\ldots$ & $\ldots$ & $\ldots$ & $16.67(06)$ & $16.20(04)$ \\
\hline
\end{tabular}

${ }^{a}$ Relative to the explosion date, MJD $=56,901.89$. 
Table 3:: Optical Photometry of SN 2014cx from LCOGT ( $1 \sigma$ Uncertainties).

\begin{tabular}{|c|c|c|c|c|c|c|c|}
\hline $\begin{array}{c}\text { UT Date } \\
(\mathrm{yy} / \mathrm{mm} / \mathrm{dd})\end{array}$ & MJD & $\begin{array}{l}\text { Phase }^{a} \\
\text { (day) }\end{array}$ & $\begin{array}{c}B \\
(\mathrm{mag})\end{array}$ & $\begin{array}{c}V \\
\text { (mag) }\end{array}$ & $\begin{array}{c}g \\
(\mathrm{mag})\end{array}$ & $\begin{array}{c}r \\
\text { (mag) }\end{array}$ & $\begin{array}{c}i \\
\text { (mag) }\end{array}$ \\
\hline 2014 Sep. 03 & 56903.130 & 1.240 & $15.32(03)$ & $15.32(01)$ & $15.23(03)$ & $15.45(02)$ & $15.54(02)$ \\
\hline 2014 Sep. 03 & 56903.390 & 1.500 & $15.34(03)$ & $15.28(03)$ & $15.16(04)$ & $15.36(03)$ & $15.51(02)$ \\
\hline 2014 Sep. 07 & 56907.770 & 5.880 & $15.12(03)$ & $15.01(03)$ & $14.93(03)$ & $14.84(02)$ & $14.92(03)$ \\
\hline 2014 Sep. 12 & 56912.685 & 10.795 & $15.10(03)$ & $14.93(02)$ & $14.96(01)$ & $14.98(03)$ & $14.98(02)$ \\
\hline 2014 Sep. 14 & 56914.755 & 12.865 & $15.20(03)$ & $14.92(03)$ & $15.07(03)$ & $14.95(03)$ & $14.92(04)$ \\
\hline 2014 Sep. 16 & 56916.690 & 14.800 & $\cdots$ & $\cdots$ & $15.00(02)$ & $14.82(02)$ & $14.93(03)$ \\
\hline 2014 Sep. 18 & 56918.685 & 16.795 & $15.25(04)$ & $14.94(05)$ & $15.03(03)$ & $14.81(02)$ & $14.92(07)$ \\
\hline 2014 Sep. 20 & 56920.545 & 18.655 & $15.30(02)$ & $14.91(02)$ & & $14.79(03)$ & $14.88(07)$ \\
\hline 2014 Sep. 23 & 56923.540 & 21.650 & $15.29(02)$ & $14.88(03)$ & $15.04(04)$ & $14.76(03)$ & $14.94(05)$ \\
\hline 2014 Sep. 27 & 56927.545 & 25.655 & $15.45(03)$ & $14.92(02)$ & $15.12(03)$ & $14.76(03)$ & $14.82(04)$ \\
\hline 2014 Sep. 29 & 56929.395 & 27.505 & $15.48(03)$ & $14.94(03)$ & & & \\
\hline 2014 Oct. 02 & 56932.520 & 30.630 & $15.58(03)$ & $14.94(02)$ & $15.20(01)$ & $14.76(01)$ & $14.81(05)$ \\
\hline 2014 Oct. 03 & 56933.715 & 31.825 & $15.56(03)$ & $14.97(03)$ & $15.29(04)$ & $14.78(02)$ & $14.79(03)$ \\
\hline 2014 Oct. 09 & 56939.690 & 37.800 & $15.73(04)$ & $15.01(03)$ & $15.34(05)$ & $14.87(03)$ & $14.80(02)$ \\
\hline 2014 Oct. 13 & 56943.830 & 41.940 & $15.80(02)$ & $15.03(02)$ & $15.39(03)$ & $14.80(02)$ & $14.85(02)$ \\
\hline 2014 Oct. 17 & 56947.475 & 45.585 & $15.86(03)$ & $15.06(02)$ & $15.40(01)$ & $14.80(02)$ & $14.79(02)$ \\
\hline 2014 Oct. 21 & 56951.565 & 49.675 & & $\cdots$ & $15.42(02)$ & $14.87(02)$ & $14.74(02)$ \\
\hline 2014 Oct. 25 & 56955.405 & 53.515 & $15.94(04)$ & $15.12(04)$ & $15.47(03)$ & $14.76(04)$ & $14.80(03)$ \\
\hline 2014 Nov. 01 & 56962.565 & 60.675 & $16.06(03)$ & $15.07(03)$ & $15.55(03)$ & $14.83(03)$ & $14.77(03)$ \\
\hline 2014 Nov. 06 & 56967.980 & 66.090 & $16.13(02)$ & $15.10(03)$ & $15.54(03)$ & $14.82(03)$ & $14.79(03)$ \\
\hline 2014 Nov. 13 & 56974.630 & 72.740 & $16.26(03)$ & $15.16(03)$ & $15.63(04)$ & $14.94(04)$ & $14.85(05)$ \\
\hline
\end{tabular}


2014 Nov. $18 \quad 56979.900 \quad 78.010 \quad 16.30(03) \quad 15.16(02) \quad 15.68(03) \quad 14.89(02) \quad 14.88(03)$ 2014 Nov. $25 \quad 56986.060 \quad 84.170 \quad 16.43(03) \quad 15.26(02) \quad 15.80(04) \quad 14.99(03) \quad 14.89(03)$ 2014 Nov. $27 \quad 56988.890 \quad 87.000 \quad 16.41(03) \quad 15.25(03)$

2014 Nov. $28 \quad 56989.780 \quad 87.890 \quad 16.49(04) \quad 15.32(03) \quad 15.83(02) \quad 14.99(02) \quad 14.97(03)$ 2014 Dec. $04 \quad 56995.820 \quad 93.930 \quad 16.65(04) \quad 15.44(02) \quad 15.96(04) \quad 15.08(03) \quad 15.00(02)$ 2014 Dec. $04 \quad 56995.850 \quad 93.960 \quad 16.71(04) \quad 15.47(03) \quad \ldots \quad \ldots$

2014 Dec. $09 \quad 57000.120 \quad 98.230 \quad 17.04(05) \quad 15.57(02) \quad 16.15(04) \quad 15.33(03) \quad 15.11(04)$ 2014 Dec. $12 \quad 57003.830 \quad 101.940 \quad 16.95(04) \quad 15.67(02) \quad 16.24(03) \quad 15.33(02) \quad 15.24(05)$ 2014 Dec. $16 \quad 57007.545 \quad 105.655 \quad 17.26(05) \quad 15.92(03) \quad 16.56(04) \quad 15.51(03) \quad 15.46(02)$ 2014 Dec. $20 \quad 57011.480 \quad 109.590 \quad 17.63(04) \quad 16.25(02) \quad 16.84(03) \quad 15.74(02) \quad 15.67(05)$ 2014 Dec. $24 \quad 57015.425 \quad 113.535 \quad 17.84(06) \quad 16.60(03) \quad 17.18(04) \quad 16.03(01) \quad 15.92(03)$ 2014 Dec. $30 \quad 57021.430 \quad 119.540 \quad 18.09(07) \quad 17.04(04) \quad 17.53(05) \quad 16.44(03) \quad 16.44(09)$

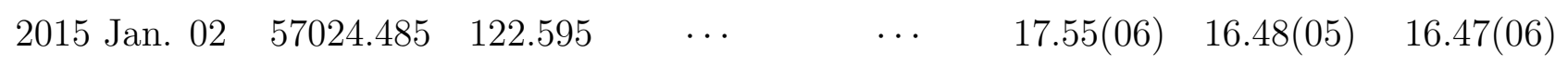
2015 Jan. $03 \quad 57025.795 \quad 123.905 \quad 18.16(05) \quad 17.04(03) \quad 17.59(04) \quad 16.48(03) \quad 16.43(05)$ 2015 Jan. $05 \quad 57027.830 \quad 125.940 \quad 18.22(05) \quad 17.14(03) \quad 17.66(05) \quad 16.51(02) \quad 16.54(03)$ 2015 Jan. $08 \quad 57030.445 \quad 128.555 \quad 18.36(05) \quad 17.21(04) \quad 17.76(04) \quad 16.59(03) \quad 16.58(03)$ 2015 Jan. $09 \quad 57031.815 \quad 129.925 \quad 18.33(05) \quad 17.19(03) \quad \ldots \quad \ldots$ 2015 Jan. $10 \quad 57032.060 \quad 130.170 \quad 18.31(05) \quad 17.15(03) \quad \ldots \quad \ldots$ 2015 Jan. $11 \quad 57033.795 \quad 131.905 \quad 18.37(05) \quad 17.28(02) \quad 17.66(03) \quad 16.64(02) \quad 16.58(02)$ 2015 Jan. $14 \quad 57036.070 \quad 134.180 \quad 18.35(05) \quad 17.23(03) \quad 17.69(04) \quad 16.63(03) \quad 16.61(04)$

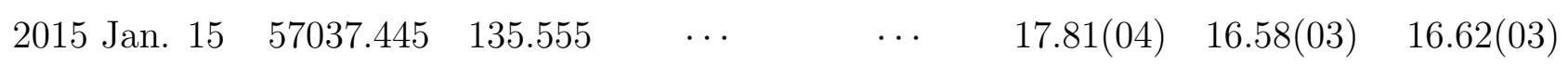
\begin{tabular}{lllllllll}
2015 & Jan. 16 & 57038.790 & 136.900 & $18.40(07)$ & $17.23(04)$ & $17.74(04)$ & $16.62(03)$ & $16.64(03)$ \\
\hline
\end{tabular}

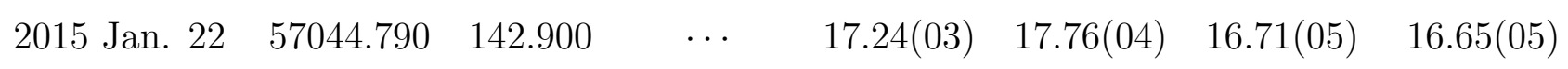
2015 Jan. $28 \quad 57050.085 \quad 148.195 \quad 18.44(07) \quad 17.32(04) \quad 17.86(05) \quad 16.69(03) \quad 16.76(05)$ 2015 Jan. $29 \quad 57051.080 \quad 149.190 \quad 18.41(08) \quad 17.33(04) \quad 17.93(06) \quad 16.72(03) \quad 16.82(05)$ 2015 Feb. $11 \quad 57064.775 \quad 162.885 \quad 18.49(08) \quad 17.52(04) \quad 17.91(04) \quad 16.79(03) \quad 16.85(04)$ 


\begin{tabular}{lllccccc} 
2015 Feb. 20 & 57073.075 & 171.185 & $18.45(08)$ & $17.57(04)$ & $\ldots$ & $\ldots$ & $\ldots$ \\
2015 June 04 & 57177.420 & 275.530 & $\ldots$ & $\ldots$ & $\ldots$ & $17.81(04)$ & $18.06(10)$ \\
2015 June 22 & 57195.790 & 293.900 & $\ldots$ & $\ldots$ & $\ldots$ & $18.03(09)$ & $18.02(06)$ \\
2015 June 30 & 57203.395 & 301.505 & $18.92(05)$ & $18.44(05)$ & $18.65(04)$ & $17.85(04)$ & $18.206(04)$ \\
2015 July 17 & 57220.260 & 318.370 & $18.93(05)$ & $18.52(04)$ & $18.69(07)$ & $18.11(06)$ & $18.200(06)$ \\
2015 Aug. 13 & 57247.330 & 345.440 & $\ldots$ & $\ldots$ & $18.70(05)$ & $18.36(05)$ & $18.259(04)$ \\
2015 Aug. 29 & 57263.255 & 361.365 & $\ldots$ & $\ldots$ & $19.02(21)$ & $18.27(23)$ & $\ldots$ \\
2015 Sep. 17 & 57282.390 & 380.500 & $\ldots$ & $\ldots$ & $19.03(05)$ & $18.50(05)$ & $18.198(05)$ \\
\hline
\end{tabular}

${ }^{a}$ Relative to the explosion date, MJD $=56,901.89$. 
Table 4: Unfiltered Photometry of SN 2014cx (1 $\sigma$ Uncertainties).

\begin{tabular}{|c|c|c|c|c|c|c|c|c|c|}
\hline MJD & Phase $^{a}$ & Mag & Error & Telescope & MJD & Phase $^{a}$ & Mag & Error & Telescope \\
\hline 56871.49 & -30.40 & $>18.76$ & & KAIT & 56949.38 & 47.49 & 14.87 & 0.08 & KAIT \\
\hline 56876.49 & -25.40 & $>19.16$ & & KAIT & 56950.31 & 48.42 & 14.73 & 0.12 & KAIT \\
\hline 56877.48 & -24.41 & $>19.06$ & & KAIT & 56952.31 & 50.42 & 14.78 & 0.06 & KAIT \\
\hline 56879.41 & -22.48 & $>18.50$ & & KAIT & 56954.33 & 52.44 & 14.79 & 0.05 & KAIT \\
\hline 56880.41 & -21.48 & $>18.67$ & & KAIT & 56957.31 & 55.42 & 14.86 & 0.05 & KAIT \\
\hline 56881.39 & -20.50 & $>18.38$ & & KAIT & 56958.33 & 56.44 & 14.84 & 0.05 & KAIT \\
\hline 56886.46 & -15.43 & $>19.48$ & & KAIT & 56959.28 & 57.39 & 14.80 & 0.07 & KAIT \\
\hline 56887.47 & -14.42 & $>19.05$ & & KAIT & 56960.26 & 58.37 & 14.88 & 0.06 & KAIT \\
\hline 56888.45 & -13.44 & $>19.22$ & & KAIT & 56964.29 & 62.40 & 14.90 & 0.07 & KAIT \\
\hline 56889.45 & -12.44 & $>19.14$ & & KAIT & 56970.32 & 68.43 & 14.80 & 0.09 & KAIT \\
\hline 56890.45 & -11.44 & $>19.13$ & & KAIT & 56971.26 & 69.37 & 14.86 & 0.07 & KAIT \\
\hline 56891.44 & -10.45 & $>19.14$ & & KAIT & 56972.26 & 70.37 & 14.80 & 0.06 & KAIT \\
\hline 56892.47 & -9.42 & $>19.26$ & & KAIT & 56976.32 & 74.43 & 14.90 & 0.07 & KAIT \\
\hline 56893.41 & -8.48 & $>19.15$ & & KAIT & 56977.25 & 75.36 & 14.85 & 0.06 & KAIT \\
\hline 56894.48 & -7.41 & $>18.88$ & & KAIT & 56978.25 & 76.36 & 14.85 & 0.12 & KAIT \\
\hline 56895.42 & -6.47 & $>19.17$ & & KAIT & 56985.23 & 83.34 & 14.89 & 0.08 & KAIT \\
\hline 56896.41 & -5.48 & $>19.22$ & & KAIT & 56986.24 & 84.35 & 14.96 & 0.06 & KAIT \\
\hline 56897.49 & -4.40 & $>19.14$ & & KAIT & 56987.23 & 85.34 & 15.09 & 0.05 & KAIT \\
\hline 56898.40 & -3.49 & $>19.13$ & & KAIT & 56988.26 & 86.37 & 15.11 & 0.08 & KAIT \\
\hline 56900.40 & -1.49 & $>19.19$ & & KAIT & 56989.19 & 87.30 & 14.99 & 0.06 & KAIT \\
\hline 56901.39 & -0.50 & $>19.11$ & & KAIT & 56990.18 & 88.29 & 15.07 & 0.05 & KAIT \\
\hline 56902.40 & 0.51 & 15.69 & 0.05 & KAIT & 56999.24 & 97.35 & 15.27 & 0.07 & KAIT \\
\hline 56903.36 & 1.47 & 15.17 & 0.07 & KAIT & 57001.22 & 99.33 & 15.28 & 0.08 & KAIT \\
\hline 56920.41 & 18.52 & 14.85 & 0.06 & KAIT & 57006.14 & 104.25 & 15.47 & 0.09 & KAIT \\
\hline 56922.33 & 20.44 & 14.65 & 0.06 & KAIT & 57015.12 & 113.23 & 16.05 & 0.07 & KAIT \\
\hline 56923.30 & 21.41 & 14.67 & 0.08 & KAIT & 57027.08 & 125.19 & 16.54 & 0.15 & KAIT \\
\hline
\end{tabular}


Table 5: UV and Optical Photometry of SN 2014cx from Swift (1 $\sigma$ Uncertainties).

\begin{tabular}{|c|c|c|c|c|c|c|c|c|}
\hline $\begin{array}{c}\text { UT Date } \\
\text { (yy/mm/dd) }\end{array}$ & MJD & $\begin{array}{l}\text { Phase }^{a} \\
\text { (day) }\end{array}$ & $\begin{array}{l}u v w 2 \\
(\mathrm{mag})\end{array}$ & $\begin{array}{l}u v m 2 \\
\text { (mag) }\end{array}$ & $\begin{array}{l}u v w 1 \\
(\mathrm{mag})\end{array}$ & $\begin{array}{c}U \\
(\mathrm{mag})\end{array}$ & $\begin{array}{c}B \\
(\mathrm{mag})\end{array}$ & $\begin{array}{c}V \\
(\mathrm{mag})\end{array}$ \\
\hline 2014 Sep. 3 & 56903.03 & 1.14 & $13.62(05)$ & $13.70(05)$ & $13.80(05)$ & $13.95(04)$ & $15.34(06)$ & $15.42(07)$ \\
\hline 2014 Sep. 4 & 56904.62 & 2.73 & $13.70(05)$ & $13.67(05)$ & $13.66(05)$ & $13.74(04)$ & $14.97(05)$ & $15.09(06)$ \\
\hline 2014 Sep. 5 & 56905.26 & 3.37 & $13.86(05)$ & $13.71(05)$ & $13.77(05)$ & $13.78(04)$ & $14.95(05)$ & $15.09(06)$ \\
\hline 2014 Sep. 6 & 56906.09 & 4.20 & $14.06(05)$ & $13.85(05)$ & $13.80(05)$ & $13.78(04)$ & $14.96(05)$ & $15.01(06)$ \\
\hline 2014 Sep. 7 & 56907.25 & 5.36 & $14.32(06)$ & $14.03(06)$ & $13.92(05)$ & $13.78(04)$ & $14.92(05)$ & $15.00(06)$ \\
\hline 2014 Sep. 8 & 56908.31 & 6.42 & $14.54(07)$ & $\cdots$ & $14.00(05)$ & $13.80(04)$ & $14.88(05)$ & \\
\hline 2014 Sep. 9 & 56909.09 & 20 & $14.80(07)$ & $14.40(06)$ & $14.11(05)$ & $13.83(04)$ & $14.92(05)$ & $14.97(06)$ \\
\hline 2014 Sep. 12 & 56912.91 & 11.02 & $15.48(09)$ & $15.19(08)$ & $14.60(06)$ & $13.99(04)$ & $14.90(05)$ & $14.97(06)$ \\
\hline 2014 Sep. 16 & 56916.28 & 14.39 & $16.22(11)$ & $16.25(10)$ & $15.30(08)$ & $14.24(05)$ & $15.02(05)$ & $14.99(06)$ \\
\hline 2014 Sep. 20 & 56920.78 & 18.89 & $17.67(24)$ & $17.80(24)$ & $16.32(11)$ & $14.62(05)$ & $15.25(06)$ & $14.96(06)$ \\
\hline 2014 Sep. 24 & 56924.71 & 22.82 & $18.21(36)$ & $18.37(42)$ & 16 . & 15.2 & 15.3 & 14 \\
\hline 2014 Sep. 28 & 56928.60 & 26.71 & $18.55(46)$ & $\ldots$ & $17.51(21)$ & $15.62(07)$ & $15.45(06)$ & $14.96(06)$ \\
\hline 2014 Oct. 4 & 56934.23 & 32.34 & $\cdots$ & $\cdots$ & $18.08(30)$ & $15.85(07)$ & $15.62(06)$ & $14.95(07)$ \\
\hline 2014 Oct. 11 & 56941.27 & 39.38 & $\cdots$ & $\cdots$ & 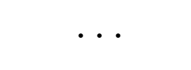 & 16.22 & $15.80(06)$ & $15.08(06)$ \\
\hline 2014 Oct. 19 & 56949.91 & 48.02 & . & $\cdots$ & $18.62(47)$ & $16.46(07)$ & $16.00(06)$ & $15.12(06)$ \\
\hline 2014 Oct. 22 & 56952.70 & 50.81 & . & $\cdots$ & $\cdots$ & $16.39(14)$ & $15.96(09)$ & $15.06(09)$ \\
\hline 2014 Oct. 24 & 56954.60 & 52.71 & $\cdots$ & $\cdots$ & $\ldots$ & $16.56(07)$ & $16.01(06)$ & $15.09(06)$ \\
\hline
\end{tabular}

${ }^{a}$ Relative to the explosion date, MJD $=56,901.89$. 
Table 6: Photometric Parameters of SN 2014cx.

\begin{tabular}{|c|c|c|c|c|c|}
\hline & $U$ & $B$ & $V$ & $R$ & $I$ \\
\hline Peak magnitude & 13.75 & 15.08 & 14.91 & - & - \\
\hline Phase of maximum $^{a}$ & 3.29 & 8.24 & 10.83 & - & - \\
\hline Plateau magnitude & - & - & 15.10 & 14.75 & 14.38 \\
\hline Decay rate $(\mathrm{mag} / 100 \mathrm{~d})$ & - & 0.42 & 0.95 & 1.09 & 1.13 \\
\hline
\end{tabular}

${ }^{a}$ Relative to the explosion date, MJD $=56,901.89$. 
Table 7: Observing Log for Optical Spectra of SN 2014cx.

\begin{tabular}{lccccc}
\hline \hline UT Date & MJD & $\begin{array}{c}\text { Phase }^{a} \\
\text { (days })\end{array}$ & $\begin{array}{c}\text { Range } \\
(\AA)\end{array}$ & $\begin{array}{c}\text { Exposure } \\
(\mathrm{s})\end{array}$ & Telescope + Instrument \\
\hline 2014 Sep. 7 & 56907.74 & 5.8 & $3400-8500$ & 2700 & LCOGT 2.0 m Telescope South + FLOYD \\
2014 Sep. 12 & 56912.69 & 10.8 & $3400-8500$ & 1800 & LCOGT 2.0 m Telescope South + FLOYD \\
2014 Sep. 18 & 56918.66 & 16.8 & $3480-8850$ & 2100 & Xinglong 2.16 m + BFOSC \\
2014 Sep. 22 & 56922.46 & 20.6 & $3400-9000$ & 1800 & LCOGT 2.0 m Telescope South + FLOYD \\
2014 Sep. 26 & 56926.71 & 24.8 & $3400-10,000$ & 1800 & LCOGT 2.0 m Telescope South + FLOYD \\
2014 Sep. 29 & 56929.65 & 27.8 & $3400-10,000$ & 1800 & LCOGT 2.0 m Telescope South + FLOYD \\
2014 Oct. 3 & 56933.70 & 31.8 & $3400-10,000$ & 2700 & LCOGT 2.0 m Telescope South + FLOYD \\
2014 Oct. 12 & 56942.46 & 40.6 & $3400-10,000$ & 2700 & LCOGT 2.0 m Telescope South + FLOYD \\
2014 Oct. 16 & 56946.60 & 44.6 & $3500-9000$ & 2100 & Lijiang 2.4 m + YFOSC \\
2014 Oct. 21 & 56951.65 & 49.8 & $3400-10,000$ & 2700 & LCOGT 2.0 m Telescope South + FLOYD \\
2014 Dec. 7 & 56998.36 & 96.5 & $3400-10,000$ & 2700 & LCOGT 2.0 m Telescope North + FLOYD \\
2015 Jan. 8 & 57030.43 & 128.6 & $3900-8800$ & 2400 & Xinglong 2.16 m + BFOSC \\
2015 July 27 & 57230.62 & 328.7 & $3900-9700$ & 900 & Gemini-North 8.1 m + GMOS \\
2015 Oct. 10 & 57305.38 & 403.5 & $3800-10,000$ & 1200 & Keck-I 10 m + LRIS \\
\hline
\end{tabular}

${ }^{a}$ Relative to the explosion date, MJD $=56,901.89$. 